OPEN ACCESS

Edited by:

Hongxi $X u$,

Shanghai University of Traditional

Chinese Medicine, China

Reviewed by:

Thomas A. Kufer,

University of Hohenheim, Germany

Wen-Qun Li,

Central South University, China

Hulya Karatas,

Hacettepe University, Turkey

${ }^{*}$ Correspondence:

Peng Yu

yu8220182@163.com

Jing Zhang

zhangjing666doc@163.com

${ }^{\dagger}$ These authors have contributed equally to this work

Specialty section:

This article was submitted to Molecular and Cellular Oncology,

a section of the journal

Frontiers in Cell and Developmental

Biology

Received: 26 October 2021 Accepted: 03 December 2021

Published: 03 January 2022

Citation:

LiZ, Chen X, Tao J, Shi A, Zhang J and Yu P (2022) Exosomes Regulate

NLRP3 Inflammasome in Diseases. Front. Cell Dev. Biol. 9:802509. doi: 10.3389/fcell.2021.802509

\section{Exosomes Regulate NLRP3 Inflammasome in Diseases}

\author{
Zhangwang $\mathrm{Li}^{1 \dagger}$, Xinyue Chen ${ }^{1 \dagger}$, Junjie $\mathrm{TaO}^{1 \dagger}{ }^{1 \dagger}$, Ao Shi ${ }^{2,3}$, Jing Zhang ${ }^{1,4 *}$ and Peng $\mathrm{Yu}^{1,5 *}$ \\ ${ }^{1}$ The Second Affiliated Hospital of Nanchang University, The Second Clinical Medical College of Nanchang University, Nanchang, \\ China, ${ }^{2}$ School of Medicine, University of Nicosia, Nicosia, Cyprus, ${ }^{3}$ School of Medicine, St. George University of London, \\ London, United Kingdom, ${ }^{4}$ Department of Anesthesiology, The Second Affiliated Hospital of Nanchang University, Nanchang, \\ China, ${ }^{5}$ Department of Metabolism and Endocrinology, The Second Affiliated Hospital of Nanchang University, Nanchang, China
}

Emerging evidence has suggested the unique and critical role of exosomes as signal molecules vector in various diseases. Numerous researchers have been trying to identify how these exosomes function in immune progression, as this could promote their use as biomarkers for the disease process and potential promising diagnostic tools. NOD-like receptor (NLR) family, pyrin domain containing 3 (NLRP3), a tripartite protein, contains three functional domains a central nucleotide-binding and oligomerization domain (NACHT), an N-terminal pyrin domain (PYD), and a leucine-rich repeat domain (LRR). Of note, existing studies have identified exosome as a novel mediator of the NLRP3 inflammasome, which is critical in diseases progression. However, the actual mechanisms and clinical treatment related to exosomes and NLRP3 are still not fully understood. Herein, we presented an up-to-date review of exosomes and NLRP3 in diseases, outlining what is known about the role of exosomes in the activation of NLRP3 inflammasome and also highlighting areas of this topic that warrant further study.

Keywords: exosomes, NLRP3, inflammasome, miRNA, diseases

\section{INTRODUCTION}

Exosomes exert a vital role in disease development (Kalluri and LeBleu, 2020). Exosomes (30-150 $\mathrm{nm}$ in diameter) arising from the membranes of multivesicular bodies (MVB) can transmit multiple biological molecules, thus regulating intercellular communication under normal and pathophysiological conditions (Pegtel and Gould, 2019). According to Vesiclepedia, a total of 349,988 proteins, 639 lipids, 27,646 messenger RNAs (mRNAs), and 10,520 microRNAs (miRNAs) have been found in exosomes. It has been revealed that exosomes could be used as a kind of disease biomarker to provide insights into the early diagnosis for cancers, such as gastric cancer, breast cancer, and prostate cancer (Jia et al., 2017; Fujita and Nonomura, 2018; Wang et al., 2018). Interestingly, the molecules in exosomes could reflect the features of the cells they come from to some extent. Compared with free biomolecules, the intrinsic features of biomolecules encapsulated by exosomes are more stable and biocompatible (Deb et al., 2021). Since exosomes were first discovered in 1983, their molecular mechanisms and functions have been increasingly explored (Zhang and Yu, 2019). It has been reported that exosomes greatly impact cancer progression, cardiovascular disease, liver fibrosis, non-alcoholic steatohepatitis (NASH), and metabolic diseases (Schroder et al., 2010; Wan et al., 20162016; Próchnicki and Latz, 2017; Moossavi et al., 2018; Tai et al., 2018; Zhou et al., 2018; Tao et al., 2020). Currently, there is a growing interest in the association between exosomes and immunoregulation mechanisms in diseases progression has been creeping up (Sharma and Johnson, 2020). For example, exosomes secreted by tumor cells have been highlighted in modulating the 
immune response in some cancer types (Greening et al., 2015). Emerging studies have confirmed that exosomes can enhance opsonization, modulate antigen presentation, and induce immune activation and immune suppression (Kalluri, 2016; Zhang and Yu, 2019).

NLRP3, a tripartite protein of the NLRP3 family of PRRs, oligomerizes to form the NLRP3 inflammasome upon activation serving as a pattern recognition receptor in the cytosol (Mangan et al., 2018; Swanson et al., 2019). NLRP3 inflammasome is widely present in immune cells. Its downstream effector proteins include caspase-1, interleukin-1 $\beta$ (IL-1 $\beta$ ), and interleukin-18 (IL-18), which exhibit a protective or detrimental role in mucosal immunity (Zhen and Zhang, 2019). Of note, emerging evidence demonstrates that thioredoxin-interacting protein (TXNIP) is of great significance to NLRP3 inflammasome activation by the TXNIP-NLRP3 interaction (Zhou et al., 2010; Lerner et al., 2012; Mohamed et al., 2014). The association between TXNIP and NLRP3 was mainly linked to reactive oxygen species (ROS) accumulation. For example, in the progression of insulin resistance-related inflammation (Strowig et al., 2012), glucose could induce TXNIP expression, and ROS triggers its dissociation from thioredoxin, thus providing a signal for NLRP3 inflammasome activation and IL-1 $\beta$ release (Willeit et al., 2018).

Emerging studies have revealed the mechanisms through which exosomes modulate NLRP3 in diverse immunity processes (Bai et al., 2019; Tavakoli Dargani and Singla, 2019; Liang et al., 2020). Though the molecular functions in diseases have been extensively researched and the field has rapidly advanced, the complete regulation of exosomes and NLRP3, as well as clinical application, are still not fully understood. This review focused on the modulation mechanisms of the NLRP3 inflammasome by exosomes and the potential treatment targets in human diseases, hoping to bring and stimulate novel insights in clinical treatment.

\section{CHARACTERISTICS AND FUNCTIONS OF EXOSOMES IN INFLAMMATION}

\section{Biogenesis and Function}

Based on size, biogenesis, and content, extracellular vesicles (EVs) secreted by cells can be grouped into three main subtypes: apoptotic bodies, microvesicles, and exosomes (Doyle and Wang, 2019). Various investigations have revealed that both exosomes and microvesicles encapsulate specific sets of proteins, lipids, and nucleic acids, which can have a marked effect on intercellular communication as molecular agents (Simons and Raposo, 2009; Ludwig and Giebel, 2012; Raposo and Stoorvogel, 2013). However, there are no effective and precise separation methods to make exosomes distinguishable from other EVs (Ludwig et al., 2019). Apoptotic bodies (800-5,000 nm in diameter) and microvesicles (200-1,000 $\mathrm{nm}$ in diameter) are directly generated through the outward budding of the plasma membrane, whereas exosomes $(30-150 \mathrm{~nm}$ in diameter) are vesicles of endocytic origin (van Niel et al., 2018). It has been reported that some vesicles (>150 $\mathrm{nm}$ in diameter) can also be secreted through an endosomal pathway and that other vesicles $(<150 \mathrm{~nm}$ in diameter) can be directly formed from the plasma membrane (Nabhan et al., 2012). Therefore, the identification of exosomes based on size must be undertaken warily.

Many studies have confirmed the role of exosomes as carriers of biomarkers for diseases. The most commonly occurring membrane proteins include tetraspanins [e.g., cluster of differentiation 63 (CD63), cluster of differentiation 9 (CD9), and cluster of differentiation 81 (CD81)], heat shock proteins (e.g., Hspa8, Hsp90), GTPases [e.g., recombinant eukaryotic translation elongation factor 1 a 1 (EEF1A1), recombinant eukaryotic translation elongation factor 2 (EEF2)], and endosomal proteins and markers (e.g., Alix) (Ibrahim and Marbán, 2016; Gemel et al., 2019). Many efforts have been made to explore the specific and functional potential of exosomes. Yang et al. measured the level of the miRNA in Alzheimer's disease and found that the serum exosomederived miR-135a, miR-193b, and miR-384 could provide novel insights for the screening and prevention of Alzheimer's disease (Yang et al., 2018a). It has also been reported that the miRNAs content of exosomes from the sputum is dysregulated in idiopathic pulmonary fibrosis (IPF), thus may be used as novel biomarkers for diagnosis (Njock et al., 2019). Moreover, exosomal signal regulatory protein a (SIRPa) proteins can effectively lead to phagocytic elimination of tumor cells (Koh et al., 2017). In prostate cancer (PC), fatty acid-binding protein 5 (FABP5) resulted as the potential significant exosomalencapsulated protein in PC patients when compared to controls (Erozenci et al., 2019). In cancer immunotherapy, exosomes have gained lots of attention because of their functional roles in regulating immune responses (Xu et al., 2020).

For its transportation, immune cell-derived exosomes could contain various cargoes, including lipids and nucleic acids that are involved in both immune processes (Chaput et al., 2005). Their release into the extracellular milieu involves the fusion of the MVB with the plasma membrane. In addition, some of the proteins carried by exosomes include the major histocompatibility complex (MHC) and costimulatory vesicles, which ultimately participate in exosome-induced regulation of immune responses. The cargoes of exosomes originate from the Golgi apparatus or the plasma membrane and are sorted into MVBs before being released as intraluminal vesicles (ILVs) (van Niel et al., 2018).

\section{Inflammation Regulation}

Over recent years, exosomes have been considered pivotal signal molecules vector in inflammatory processes that transfer proteins, lipids, and nucleic acids, thus influencing the target cell's metabolism in many diseases, including cancer, cardiovascular disease, and neurodegenerative disorder (Zhang et al., 2019a; Console et al., 2019; Zhou et al., 2020a; Bouchareychas et al., 2020). For example, exosome-mediated delivery of miR-155, miR-124-3p, miR-138 are involved in acute lung inflammation, traumatic brain injury, and endometriosis, respectively (Huang et al., 2018; Zhang et al., 2019b; Jiang et al., 2019). It has been found that the liposome-like nanoparticles (LLNs) assembled with lipids from Ginger ELNs 
(GELNs) are of significance in intestinal stem cells (Ju et al., 2013). Meanwhile, it has been reported that lipid carried by exosomes may help to reinstate lipid raft functions and restore barrier integrity in inflammatory bowel disease (Bowie et al., 2012). Experiments associated with exosomal-encapsulated proteins in inflammation have also been conducted. Macrophage exosomes can deliver protein to the inflamed brain, thus affecting inflammation (Yuan et al., 2017). S100 Calcium Binding Protein A9 (S100-A9) protein in exosomes can promote inflammation in polycystic ovary syndrome ( $\mathrm{Li}$ et al., 2020). It has been noted that interleukin-35 encapsulated by exosomes may be a critical molecular for suppressing inflammatory responses (Kang et al., 2020).

Recently, there have been more and more studies on the regulation of NLRP3 by exosomes inflammatory mechanisms (Bai et al., 2019; Liang et al., 2020; Si et al., 2021). For example, Yang et al. found that umbilical cord mesenchymal stem cellsderived exosomes (UMSC-Exo) attenuated production of cleaved caspase- 1 and subsequently decreased IL- $1 \beta$ and IL-18 release and pyroptosis. circHIPK3 released by UMSC-Exo downregulated miR-421, resulting in increased expression of fork head box class $\mathrm{O}$ 3a (FOXO3a), which could inhibit NLRP3 activation (Yan et al., 2020). Herein, we discussed the relationship between the exosomes and NLRP3.

\section{CHARACTERISTICS AND PATHOLOGICAL FUNCTIONS OF NLRP3 INFLAMMASOME}

NLR protein family has 22 members in humans and at least 34 members in rodents (Ting et al., 2008). As a unique receptor, NLRP3 is the best characterized and the most extensively studied among the NLRP3 family (Yang et al., 2019). NLRP3 consists of three functional domains: an amino-terminal PYD, a NACHT, and a LRR (Swanson et al., 2019). Accumulating evidence has reported that NLRP3 inflammasome has a critical role in inflammatory diseases (Mangan et al., 2018).

NLRP3 inflammasome is a high-molecular-mass protein complex consisting of an upstream sensor protein NLRP3, a downstream effector protein caspase-1, and an apoptosisassociated speck-like protein containing a CARD (ASC) (Lamkanfi and Dixit, 2012). Of note, NIMA related kinase7 (NEK7), a newly demonstrated NLRP3-binding protein, regulates NLRP3 oligomerization and activation by forming a supramolecular NLRP3-NEK7 complex (He et al., 2016a). ASC has two domains, containing PYD and C-terminal caspase recruitment domain (CARD), which can interact with other proteins (Lu et al., 2016). ASC combines with NLRP3 by homotypic PYD-PYD interactions, after which it recruits procaspase-1 through CARD-CARD interactions forming the NLRP3 inflammasome (Cai et al., 2014; Sharif et al., 2019). Then, the dormant pro-caspase-1 is cleaved into active caspase-1, which trims pro-IL-1 $\beta$ and pro-IL-18 to generate mature and active cytokines IL-1 $\beta$ and IL-18 (Mangan et al., 2018). Caspase-1 also cleaves pro-gasdermin-D (GSDMD) to generate an N-terminal fragment, after which it forms GSDMD pores, controlling the release of IL- $1 \beta$ and IL-18
(Rathinam et al., 2019). This enables cytokine secretion, water influx, osmotic swelling, and cell ruptures, eventually leading to pyroptosis (Liu et al., 2016; Orning et al., 2019; Liu et al., 2020).

To the best of our knowledge, typically in most cell types, the activation of NLRP3 occurs requiring a minimum of two steps: Priming and Activation (Xue et al., 2019a; Wang and Hauenstein, 2020). Priming is the first step initiated by various damage associated molecular patterns (DAMPs) and pathogenassociated molecular patterns (PAMPs) that are recognized by Toll-like receptors (TLRs), activating the nuclear factor $\kappa \mathrm{B}$ (NF$\kappa B$ ) signaling pathways (Gritsenko et al., 2020). NF- $\kappa B$ could enhance the transcription of pro-IL-1 $\beta$, pro-IL-18, and NLRP3 (Afonina et al., 2017). Furthermore, the second step (activation) is the oligomerization of NLRP3 and the assembly of NLRP3, NEK7, ASC, and pro-caspase-1 into the NLRP3 inflammasome (Strowig et al., 2012), which is mostly triggered by adenosine triphosphate (ATP) (Karmakar et al., 2016), purinergic ligand-gated ion channel 7 receptor (P2X7R) (Wang et al., 2020), mitochondrial reactive oxygen species (mtROS) (Zhong et al., 2016), mitochondrial damage (Zhong et al., 2016), autophagic dysfunction (Xue et al., 2019b), dynamin-related protein 1 (Drp1) (Wang et al., 2014), oxidized mitochondrial DNA (ox-mtDNA) (Jia et al., 2020a), mitochondrial abtiviral-signaling protein (MAVS) (Subramanian et al., 2013) and so on (Tschopp and Schroder, 2010; Seoane et al., 2020).

Currently, numerous studies have revealed that NLRP3 inflammasome has a crucial role in the pathogenesis of many diseases (Koka et al., 2017; Huang et al., 2021), such as cancer (Bruchard et al., 2013), cardiovascular diseases (Liu et al., 2018a), and metabolic diseases (Sharma and Kanneganti, 2021). NLRP3 inflammasome activation is induced by three different pathways in different cells, including canonical pathway, non-canonical pathway, and alternative pathway (Figure 1).

\section{EXOSOME AND NLRP3 INFLAMMASOME ACTIVATION PATHWAYS}

The crosstalk between exosome and NLRP3 inflammasome has been demonstrated with converging evidence in recent years. The inhibition of exosomal programmed death-ligand 1 (PD-L1) can result in systemic anti-tumor immunity (Poggio et al., 2019). Exosome-signal regulatory protein $\alpha(\mathrm{SIRP} \alpha)$ can interact with cluster of differentiation 47 (CD47) exiting in the tumor cells surface, thus restricting macrophages from engulfing tumor cells (Koh et al., 2017). Several experiments have revealed that exosomes can modulate immunity in diseases by monitoring programmed cell death-1 (PD1), gp130/STAT3 signaling, and the TLR4/NF-кB/NLRP3 inflammasome (Yang et al., 2018b; Ham et al., 2018; Dai et al., 2020). An increasing body of evidence reveals that exosomes are involved in the occurrence and development of diseases through NLRP3 inflammasome canonical and non-canonical pathways (Shi et al., 2020; Cai et al., 2021). Remarkably, emerging studies have uncovered the potential regulatory role of NLRP3 inflammasome activation on exosome production. Based on the available evidence, exosomes were demonstrated to exert dual effects on inflammasome activation (Noonin and Thongboonkerd, 2021). Promotion of 


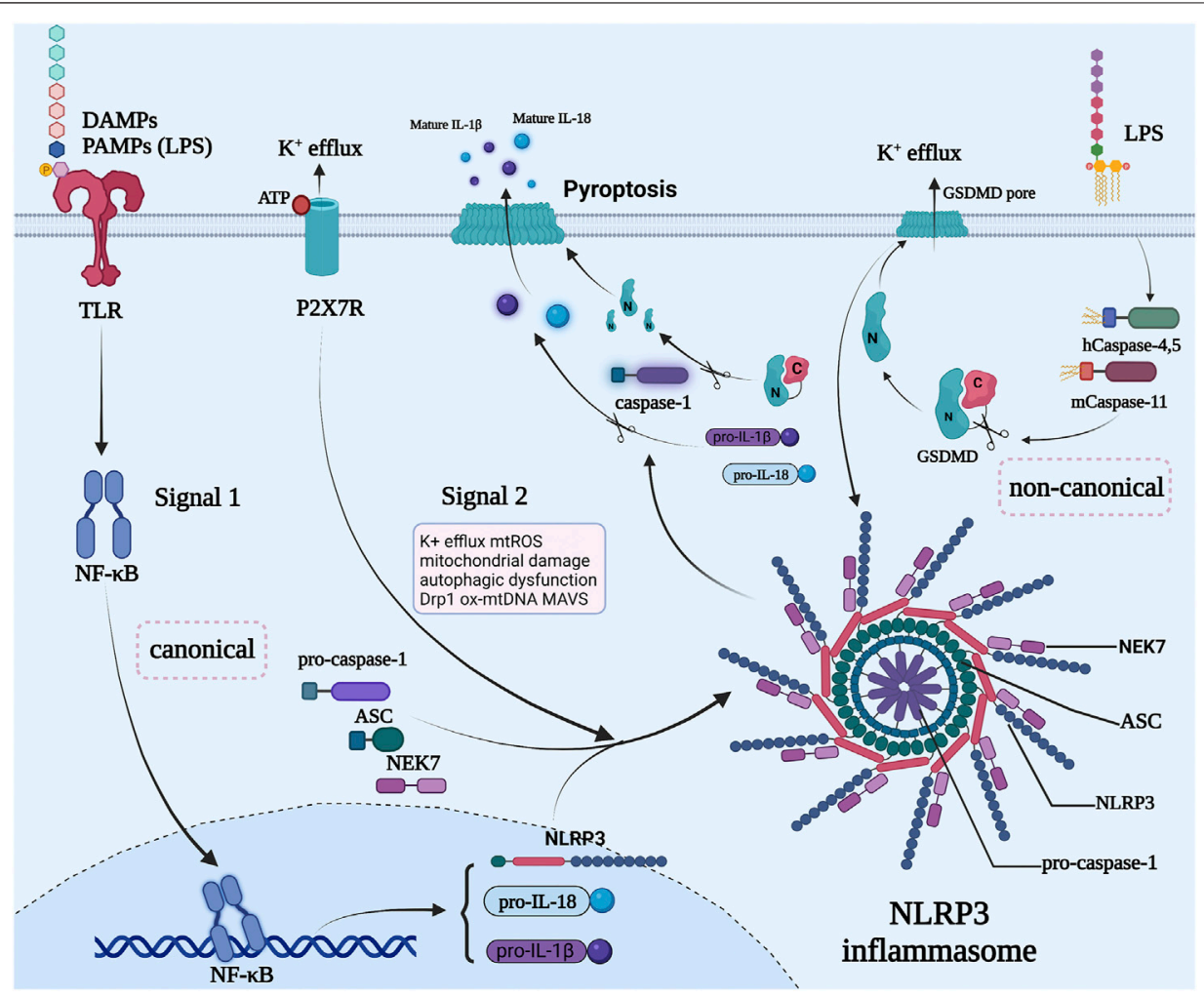

FIGURE 1 | Canonical and non-canonical NLRP3 inflammasome pathways. NLRP3 inflammasome is activated through a process, which involves a minimum of two steps in most cell types. First, a priming step is required by treating cells with a toll-like receptor ligand like lipopolysaccharides (LPS), which activates the NF- $\mathrm{B}$ pathway to induce the transcription of NLRP3, caspase-1, and pro-IL-1 $\beta$. Signal 2 is provided by PAMPs or DAMPs that activate multiple events mainly induced by potassium efflux. The inflammasome is formed by assembling NLPR3, NEK7, ASC, caspase-1. Next, the activated NLRP3 inflammasome acts as a platform for the processing of caspase-1, whose main function is the conversion of the inactive cytokines pro-IL-1 $\beta$, pro-IL-18, and GSDMD into their active forms. Non-canonical NLRP3 inflammasome activation is triggered by the cytosolic LPS sensing by caspase 4/5/11 and then they cut their substrates GSDMD, resulting in the formation of GSDMD membrane pores, causing potassium efflux, which promotes the activation of NLRP3 inflammasomes. The activated NLRP3 inflammasome further cuts the GSDMD to form more membrane pores and induce the maturation of pro-IL-1 $\beta$, pro-IL-18, and caspase-1, causing pyroptotic cell death. The figure was constructed with BioRender (https://biorender.com/).

inflammation (Liang et al., 2020) or control of pathogenesis (Singla et al., 2019) can have both positive and negative aspects, which depend on the molecular composition of exosomes (Figure 2). Therefore, a better understanding of the relationship between exosome and NLRP3 inflammasome activation may reveal a potential target for the therapy of NLRP3 inflammasome-related diseases.

\section{Exosomes and the Canonical NLRP3 Activation Pathway}

Previous studies have reported that NLRP3 inflammasome is activated by a minimum of two steps in most cell types (Sutterwala et al., 2006; Bauernfeind et al., 2009). First, a priming step is required by treating cells with TLR ligands, DAMPs and PAMPs like LPS, which activates the NF- $\kappa B$ pathway to induce the transcription of NLRP3, caspase-1 $\beta$, and pro-IL-18 (Sutterwala et al., 2006; Lu et al., 2014). This is followed by treating cells with "danger signal" molecules, such as mtROS, mitochondrial damage, autophagic dysfunction, Drp1, ox-mtDNA, MAVS, and so on (Elliott and Sutterwala, 2015; He et al., 2016b; Jo et al., 2016; Zhou et al., 2020b; Weber et al., 2020), which leads to multiple signaling events mainly induced by potassium efflux. These events trigger the assembly of NLRP3 inflammasome (Cai et al., 2014) that manifests as a large perinuclear ASC speck in the cell (Masumoto et al., 1999). Next, the activated NLRP3 inflammasome acts as a platform for the processing of caspase-1. After processing, the pro-caspase1 becomes the functional caspase- 1 , whose main function is the conversion of the inactive and intracellularly potent proinflammatory cytokines pro-IL-1 $\beta$, pro-IL-18, and the poreforming protein GSDMD) into their active forms (Miao et al., 2006; Jo et al., 2016). Mature IL-1 $\beta$ and IL-18 are then secreted from the cells (Dinarello, 2009), leading to pyroptosis. Emerging evidence has shown that exosomes can affect the key molecules in the canonical pathway by secreting different substances, which in turn affects the process of NLRP3 inflammasome-related disease (Yan et al., 2020; Li et al., 2021; Si et al., 2021; Wang et al., 2021). Dai et al. found that miR-148a enhanced cell viability and relieved cardiac enzymes dysregulation and $\mathrm{Ca} 2+$ overload in ischemia/ reperfusion-induced neonatal rat cardiomyocytes (NCMs). M2exos-carried miR-148a could directly target TXNIP and obviously 


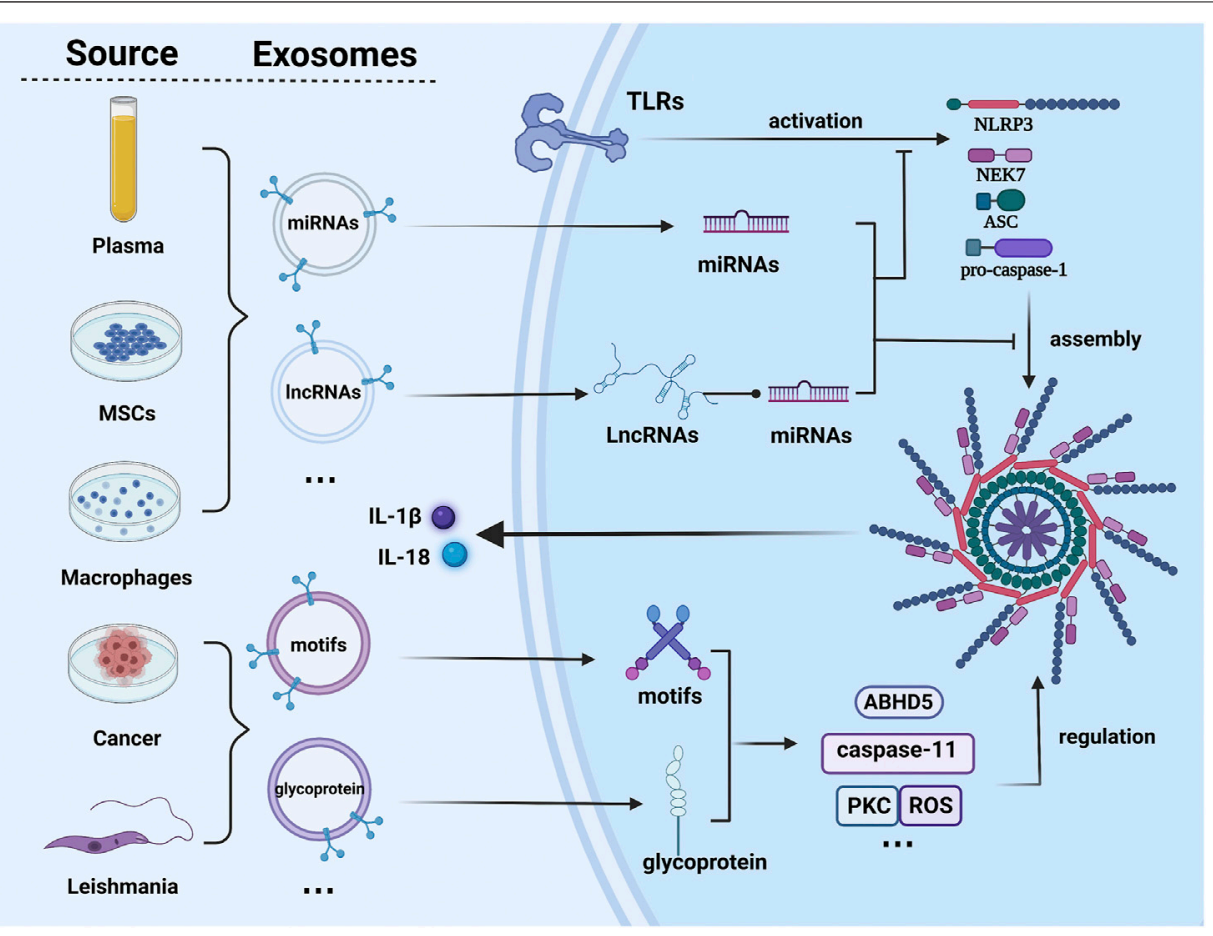

FIGURE 2 |Different sources-derived exosomes regulate the NLRP3 pathway in various patterns. Exosomes from plasma, macrophages, and mesenchymal stem cells suppress the activation of the NLRP3 pathway or the expression of the key molecule from the related signaling axis to regulate the progression of diseases. Otherwise, cancer cells and some pathogens may behave in another way. They release particular exosomal antigens and morbid substances to model a favorable environment for their own survival. Several pathogenic patterns were found. IncRNA, long non-coding RNA; miRNA, microRNA; PKC, Protein Kinase C. The figure was constructed with BioRender (https://biorender.com/).

inhibited the expression of TXNIP, significantly inhibiting TLR4 expression. The downstream NF- $\mathrm{BB}$ activation and NLRP3 inflammasome components expression, therefore, were markedly suppressed, which alleviated myocardial ischemia/ reperfusion injury (Dai et al., 2020). Meanwhile, Yan et al. reported that circular homeodomain-interacting protein kinase 3 (circHIPK3) from UMSC-Exo can downregulate miR-421, resulting in the upper expression of FOXO3a, thus preventing the activation of NLRP3 inflammasome and caspase- 1 prevent pyroptosis and repairing ischemic muscle injury (Yan et al., 2020).

\section{Exosomes and the Non-Canonical NLRP3 Activation Pathway}

Unlike the canonical pathway, NLRP3 inflammasomes can also be activated by Human caspase- 4,5 or Murine caspase- 11 directly sensing the cytosolic LPS from Gram-negative bacteria (Shi et al., 2014), then the caspase-4/5/11 could then cut their substrate GSDMD, resulting in the formation of GSDMD membrane pores (Ding et al., 2016). Furthermore, potassium efflux caused by GSDMD membrane pores promotes the activation of NLRP3 inflammasomes. The activated NLRP3 inflammasomes further cut the GSDMD to form more membrane pores and induce the maturation and secretion of IL-1 $\beta$, IL-18, and caspase-1, eventually causing pyroptotic cell death (Bossaller et al., 2012; Antonopoulos et al., 2015; Komada and Muruve, 2019). According to the literature, there are no reports of studies noted that exosomes could regulate the non-canonical NLRP3 inflammasome activation pathway to influence the development of diseases. However, the non-canonical NLRP3 inflammasome activation pathways exert significant clinical ramifications (Downs et al., 2020), thus more studies are urgently needed to uncover the mechanisms underlying the regulation of exosomes on the non-canonical NLRP3 inflammasome activation to explore potential therapeutic targets.

Figure 1 Different sources-derived exosomes regulate the NLRP3 pathway in various patterns. Exosomes from plasma, macrophages, and mesenchymal stem cells suppress the activation of the NLRP3 pathway or the expression of the key molecule from the related signaling axis to regulate the progression of diseases. Otherwise, cancer cells and some pathogens may behave in another way. They release particular exosomal antigens and morbid substances to model a favorable environment for their own survival. Serval pathogenic patterns were found. PKC, Protein Kinase C; miRNA, microRNA; lncRNA, long non-coding RNA.

\section{NLRP3 Downstream Exosomes Release-Inflammation and More Than Inflammation}

The activation of the NLRP3 inflammasome could result in the maturation and exosomal release of IL-1 $\beta$, IL-16, and ASC (Qu et al., 2009; Dubyak, 2012), thus exosomes exert a critical 
TABLE 1 | Source of exosomes and roles in regulation of NLRP3-related diseases.

\begin{tabular}{|c|c|c|c|c|}
\hline Disease & Exosomes source & Pivotal molecules & Role of the exosomes & Ref. \\
\hline \multirow[t]{3}{*}{ Cancer } & Lung cancer cells & TRIM59 & TRIM59/ABHD5/NLRP3 signaling axis & Lerner et al. (2012) \\
\hline & M2 macrophages & miR-223 & $\begin{array}{l}\text { Repressing the canonical NF-kB and TLR-9- NLRP3 } \\
\text { pathway }\end{array}$ & $\begin{array}{l}\text { Becatti et al. (2012), Mao } \\
\text { et al. (2019) }\end{array}$ \\
\hline & T24 cells, SV-HUC-1 cells & miR-375-3p & A bladder cancer suppressor via Wnt/ $\beta$-catenin pathway & Vandanmagsar et al. (2011) \\
\hline \multirow{5}{*}{$\begin{array}{l}\text { Ischemia/Reperfusion } \\
\text { injury }\end{array}$} & Mesenchymal stem cells & miR-320b & Inhibitor of the expression of NLRP3 gene & Elliott and Sutterwala (2015) \\
\hline & Plasma & $\mathrm{miR}-148 \mathrm{a}$ & $\begin{array}{l}\text { Reduction of myocardium damage via TXNIP/NLRP3/ } \\
\text { caspase-1 pathway }\end{array}$ & Saberi et al. (2009) \\
\hline & Mesenchymal stem cells & LncRNA KLF3-AS1 & $\begin{array}{l}\text { Sponge of miR-138-5p, which can overexpress SIRT, } \\
\text { then inhibit NLRP3 }\end{array}$ & Weber et al. (2020) \\
\hline & Umbilical cord stem cells & $\operatorname{miR}-26 b-5 p$ & $\begin{array}{l}\text { Suppressing the polarization of M1 macrophages leading } \\
\text { to the downregulation of TLR-2,4,6 }\end{array}$ & Zhang et al. (2021a) \\
\hline & Adipose stem cells & Unidentified miRNAs & Inhibition of Wnt/ $\beta$-catenin signaling pathway & Zhang et al. (2019c) \\
\hline Myocardial infarction & Umbilical cord stem cells & $\operatorname{miR}-100-5 p$ & $\begin{array}{l}\text { Downregulation of FOXO3 translation to block NLRP3 } \\
\text { activation }\end{array}$ & Incalza et al. (2018) \\
\hline $\begin{array}{l}\text { Carotid endarterium } \\
\text { injury }\end{array}$ & Adipose stem cells & Pre-STC1 & Low-expression of NLRP3 inflammasome & Pajukanta et al. (2004) \\
\hline Dox cardiomyopathy & Embryonic stem cells & $\begin{array}{l}\text { Anti-inflammation } \\
\text { cytokines }\end{array}$ & $\begin{array}{l}\text { Inducing M2 macrophages polarization and releasing of } \\
\mathrm{IL}-10\end{array}$ & Gritsenko et al. (2020) \\
\hline \multirow[t]{3}{*}{ Diabetes } & Mesenchymal stem cells & $\mathrm{miR}-126$ & $\begin{array}{l}\text { Downregulation of HMGB1, which can stimulate TLR4- } \\
\text { NLRP3 }\end{array}$ & Zhang et al. (2021b) \\
\hline & Adipose stem cells & Unidentified miRNAs & Inhibitor of ROS-TXNIP-NLRP3 pathway & Wang et al. (2021) \\
\hline & Pericytes, endotheliocytes & circEhmt1 & $\begin{array}{l}\text { Regulation of high glucose microvascular dysfunction via } \\
\text { the NFIA/NLRP3 pathway }\end{array}$ & Bechard et al. (2020) \\
\hline Atherosclerosis & Plasma & $\mathrm{miR}-223$ & Inhibitor of the expression of NLRP3 gene & Xue et al. (2019a) \\
\hline Pulmonary fibrosis & Endometrial stem cells & miRNA Let-7 & $\begin{array}{l}\text { Regulating mtDNA damage, repressing LOX1/NLRP3/ } \\
\text { caspase } 3\end{array}$ & Zhang et al. (2021c) \\
\hline \multirow[t]{2}{*}{ Leishmaniasis } & Leishmania & GP63 & $\begin{array}{l}\text { Influencing HZ-induced NLRP3 pathway activation and } \\
\text { cleaving the inflammasome complex }\end{array}$ & Ridker et al. (2017) \\
\hline & Leishmania & LPG & Involving in activation of non-canonical pathway of NLRP3 & Minutoli et al. (2016) \\
\hline HIV-1 & $\begin{array}{l}\text { Human bone marrow derived } \\
\text { macrophages }\end{array}$ & HIV protein Nef & Redistributing TLR4 toward lipid rafts & Tang et al. (2020) \\
\hline $\begin{array}{l}\text { Gastrointestinal } \\
\text { helminth }\end{array}$ & Worms & micro-RNAs & $\begin{array}{l}\text { Enhancing the NLRP3-dependent IL-18 and IL-1 } \beta \\
\text { secretion }\end{array}$ & Kore et al. (2021) \\
\hline Sepsis & M1 and M2 macrophages & miR-93-5p & Targeting on TXNIP and influence the activation of NLRP3 & Haneklaus et al. (2013) \\
\hline Muscle ischemia & Umbilical cord stem cells & miR-29b & $\begin{array}{l}\text { Binding to cPWWP2A and regulate the PWWP2A/Rb1/ } \\
\text { AMPKa2/NLRP3 signaling pathway }\end{array}$ & Bruchard et al. (2013) \\
\hline Parkinson's disease & Adipose stem cells & $\operatorname{miR}-188-3 p$ & Suppression of NLRP3 and CDK5 & Zhang et al. (2020) \\
\hline
\end{tabular}

TRIM59, tripartite motif-containing 59; ABHD5, abhydrolase domain containing 5; NLRP3, NLR family protein containing a pyrin domain 3; NF- $\kappa B$, nuclear factor $\kappa B$; TLR, toll-like receptor; SV-HUC-1 cells, human bladder cell biochemistry pillon; Wnt/ $\beta$-catenin, wingless/ $\beta$-catenin; LncRNA, long non-coding RNA; SIRT, sirtuin; FOXO3, forkhead box O3; STC-1, stanniocalcin-1; IL, interleukin; HMGB1, high-mobility group protein 1; ROS, reactive oxygen species; TXNIP, thioredoxin-interacting protein; NFIA, a transcription factor; mtDNA, mitochondrial RNA; LOX1, lectin-type oxidized LDL receptor 1; AMPKa2, AMP kinase 2 ; CDK5, cyclin dependent kinase 5.

role in forming the pro-inflammatory status in tissues. However, the NLRP3-promoted exosomes secretion seems not limited to loading inflammatory cytokines. A recent review of exosomes in helping a-synuclein transmission in Parkinson's disease (PD) suggests that NLRP3 could influence diseases not only by inducing inflammatory status and pyroptosis but in a way of facilitating the exosomal release of specific pathological proteins (Si et al., 2021). The elevated membrane-shed exosomes could even load protective molecules and chemo-active compounds, like nitric oxide aganist cardiovascular damage (Pearce et al., 2021). Therefore, exosomes exert as "a double-edged sword" in inflammation.

NLRP3 regulating exosomes in diseases can be interestingly affected by a wide range of chemokines. Sarkar et al. found that $\mathrm{Mn}^{2+}$ can induce cell-to-cell transfer of the inflammasome adaptor protein ASC in exosomes, which might be a potential mechanism in neurodegenerative diseases (Sarkar et al., 2019).
Carbon monoxide can activate the NLRP3 pathway and the secretion of exosomes (Chen et al., 2021). Betaine inhibits the extracellular secretion of secretory lysosomes containing IL- $1 \beta$, then inhibits the extracellular secretion of IL- $1 \beta$-containing exosomes, thereby inhibiting IL $-1 \beta$ release (Xia et al., 2018).

\section{THE REGULATORY MECHANISMS OF EXOSOMES TO NLRP3 INFLAMMASOME IN DISEASES}

NLRP3 inflammasomes act as a platform for activating cytokines like IL-1 $\beta$, and is a key meditator of the inflammatory response (Zamboni and Lima-Junior, 2015). In host-response and pathogens resistance, the inflammasome-induced response has been proven to be of great importance. Moreover, the modern lifestyle has brought new challenges to healthcare, including the problem of chronic diseases hallmarks. The reprogramming of 
innate immune cells and persistent activation of inflammasomes have been identified as essential in the mechanisms underlying an array of non-communicable diseases, thus making inflammasome a promising target for therapeutic use.

The model of NLRP3 inflammasome activation varies among different types of diseases. Potassium efflux and NEK7, which directly interact with NLRP3 and assist its activation, seem to be the conserved elements in NLRP3 activation (He et al., 2016a; Shi et al., 2016). Exosomes participate in the regulation of the whole process of NLRP3 activation. It has been reported that exosomes can carry the antigens or non-coding RNAs, which in turn could influence the expression of some key molecules (Liu et al., 2018b; Cai et al., 2021). For example, stem cells-derived exosomes can reverse the immune cell reprogramming and inflammatory state by correcting the over-activation of NLRP3. In addition, exosomes which targeting NLRP3 and its downstream IL-1 $\beta$ offer an anti-inflammation therapy method without increasing the risk of infection, as anti-IL-1 $\beta$ therapy might directly induce immunosuppression (Ridker et al., 2017). The summary of current exosomal NLRP3 regulation in diseases is presented in Table 1.

\section{Exosomal Anti-Pyroptosis via NLRP3 Inflammasome in Cardiovascular Diseases}

Pyroptosis is a newly founded cell death process characterized by excessive inflammatory cytokines release, which closely associated with the NLRP3-GSDMD pathway. In ischemia/ reperfusion (I/R) injury models, NLRP3, caspase-1, and GSDMD are notably heightened (Jia et al., 2020b). It has been reported that mitochondrial dysfunction and ROS accumulation are crucial in I/R-related NLRP3 activation (Minutoli et al., 2016; Gong et al., 2018; Jia et al., 2020b).

Of note, human bone marrow mesenchymal stem cell (MSC)derived dramatically suppresses the expression of NLRP3 in the I/R myocardium. Tang et al. discovered that MSC exosomal miR$320 \mathrm{~b}$ could directly target the NLRP3 molecule, then negatively regulate the downstream expression of the caspase-1, thus inhibiting the pyroptosis in the rat myocardial $\mathrm{I} / \mathrm{R}$ model (Tang et al., 2020). Results of recent proteomics research between infract and pre-infract myocardium provided support for the protective function of human MSC-derived exosomes against cell pyroptosis (Kore et al., 2021). Damage to the ischemic myocardium can be reduced by exosomal miR-148a via inhibiting the TXNIP-NLRP3-caspase-1 pathway (Becatti et al., 2012). Sirtuin 1 (SIRT1) is a potent protector from aging-associatedpathologies. Furthermore, SIRT1 was identified at a critical point in exosomal NLRP3 regulation. Long non-coding RNA (lncRNA) KLF3-AS1, a molecular sponge of miR-138-5p, was discovered at a vital point in exosomal NLRP3 regulation (Mao et al., 2019). Highly concentrated miR-138-5p can upregulate the expression of SIRT1, which has a critical role in NLRP3 auto-inhibition, thus decreasing the myocardial infarction-induced damage (Mao et al., 2019).

Over recent years, many studies reported that doxorubicin (DOX) cardiotoxicity could be alleviated through embryonic stem cell-derived exosomes (ES-Exo) therapy. Pyroptosis and inflammation have been found to be linked to DOX-induced cardiac remodeling and cell death, which can be alleviated by ESExo (Tavakoli Dargani and Singla, 2019). Singla and others first demonstrated that ES-Exo-induced M2 macrophages polarization and subsequent TLR4-NLRP3 downregulation were involved in exosomal ameliorating DOX-induced pyroptosis (Singla et al., 2019). Yet, more specific data on the underlying mechanism are warranted.

\section{Exosomes-Meditated NLRP3 Sensing in Metabolic Diseases}

Previous studies have suggested that NLRP3 inflammasome does not merely work as a pro-inflammatory mediator but also as an unexpected sensor for metabolic stress. Cholesterol accumulation can give the priming signal for NLRP3 activation (Vandanmagsar et al., 2011). By directly interacting with TLR4, subsequently formed cholesterol crystal can also stimulate the NLRP3 inflammasome activation afterward (Saberi et al., 2009). The persistent activation of NLRP3 results in chronic inflammation, one of the key mechanisms in various metabolic diseases, which could be alleviated by ES-Exo. As a vector of intercellular genetic exchange, ES-Exo can downregulate the chronic NLRP3 activation at the cellular expression level, thus acting as a promising therapy carrier toward long-term inflammatory states.

Hyperglycemia-induced retinal inflammation and diabetic osteoporosis can be alleviated by ES-Exo (Zhang et al., 2021a). Previous studies have indicated that MSC-Exoderived miR-126 can suppress hyperglycemia-induced inflammation by down-regulating high mobility group box-1 (HMGB1) protein, which can bind to TLR2, TLR4, and inflammasomes then help activate the NLRP3 pathway (Zhang et al., 2019c). HMGB1 is actively generated in human retinal endothelial cells and released after retinal damage or pathogenic changes. Diabetic limb ischemia is characterized by continuous ischemia and hyperglycemia in the muscle microenvironment. In addition, constant metabolic disorders and hyperglycemia in the tissue are expected to result in inflammation and oxidative stress in the tissue (Incalza et al., 2018).

TXNIP was at first in metabolism diseases identified as a key molecule of NLRP3 (Pajukanta et al., 2004). However, more recently published studies showed that TXNIP is extensively related to varieties of chronic inflammation in categories of different diseases, such as Alzheimer's diseases and pancreatic cancer (Bechard et al., 2020; Zhang et al., 2021b), which suggests that TXNIP might be a promising vital meditator in the complex signal conduction network among oxidative stress, mitochondrial stress, ROS accumulation, and NLRP3 inflammasome-induced pyroptosis. Zhang et al. found that adipose-stem-cells-derived exosomes can improve endothelial cells function in diabetic limb ischemia. The inhibition of the ROS-TXNIP-NLRP3 pathway by miRNAs derived from exosomes was inferred as a major inner mechanism; however, further sequencing of miRNAs derived from the exosomes is needed (Zhang et al., 2021c). 
Hyperlipidemia is a significant risk factor inducing atherosclerosis. Paeonol, as a promising drug for atherosclerosis, can raise the expression level of plasmaderived exosomal miRNA-223 in rats (Shi et al., 2020). miRNA-223 is an inhibitor of NLRP3 inflammasomes forming, whose upregulation can notably delay the progression of chronic inflammation in atherosclerosis (Haneklaus et al., 2013).

\section{Cancer-Derived Exosomes in Orchestrating the Polarization of TAMs Through NLRP3}

Metabolic reprogramming of tumor-associated macrophages (TAMs) has a terminal role in cancer progression and resistance (Zhang et al., 2020). There is increasing evidence suggesting that exosomes, as a novel cellular communication mechanism, are engaged in malignant cells orchestrating tumor microenvironment (TME) via TAMs. cancer-derived exosomes can reportedly cause polarization in TAMs, thus increasing IL-1 $\beta$ secretion toward the microenvironment (Atay et al., 2011; Bardi et al., 2018; Li et al., 2018), which is related to poor clinical prognosis. The heightened exosome-induced IL- $1 \beta$ secretion facilitates the formation of a pro-inflammatory state in TME, which has been found among various solid neoplasms in previous studies (Oh et al., 2016; Wu et al., 2016; DmitrievaPosocco et al., 2019). Immune cells secreting IL-1 $\beta$ depend on the activation of NLRP3 inflammasomes. Interestingly, both cancer progression and lung metastasis can be exhibited by ultraviolet-irradiated apoptotic cancer cells through enhancement of the PPAR $\gamma /$ PTEN signaling in macrophages (Kim et al., 2019). Thus, the effects induced by cancer cells-derived exosomes are heterogeneous.

TAMs are highly plastic and can rapidly adapt to microenvironmental changes (Andrejeva and Rathmell, 2017). They sensitively detect the signals transported by exosomes and then genetically reprogram their function and phenotypes. NLRP3 pathway widely exists in innate immune cells and is highly expressed in immunosuppressive and inflammatory microenvironments. Meanwhile, cancer cells can directly or indirectly affect the key molecules in the NLRP3 pathway, thus leading TAMs reprogramming toward a proinflammatory state. Liang et al. reported murine lung cancerderived exosomes, which express tripartite motif-containing 59 (TRIM59) and can regulate the NLRP3 pathway, intermediated by upregulating abhydrolase domain containing 5 (ABHD5) (Liang et al., 2020). TRIM59 directly induces the ubiquitination of ABHD5, leading to its proteasomedependent degradation, activating the NLRP3 inflammasome signaling pathway, and promoting the secretion of IL-1 $\beta$ by macrophages; As the final result, exosomal TRIM59 facilitates lung cancer growth and metastasis. Besides, ABHD5 is an important regulator in lipid metabolism, whose deficiency has been shown to be a permission signal of the reprogramming of macrophages and the activation of the NF- $\kappa \mathrm{B}$-dependent NLRP3 inflammasomes pathway (Shang et al., 2019).
The polarization of TAMs exerts a role in cancer progression. M1-like TAMs have a tendency toward forming proangiogenic and pro-inflammatory states (Chen et al., 2011), and M2-like TAMs tend to engage in tissue remodeling. It has been reported that miRNA-223 can mediate the inflammatory response in macrophages by suppressing the canonical NF- $\mathrm{BB}$ pathway, which is related to the expression of NLRP3 (Liu et al., 2015). Endogenous miRNA-223 can be transported by exosomes toward hepato-carcinoma cells to inhibit tumor proliferation (Aucher et al., 2013). Based on this, it has been suggested that exosomes may serve as a potential therapeutic target among various cancers. In addition, with a current hypothesis, further studies are needed to validate the precise mechanism of exosome-mediated-NLRP3 inflammasome activities in cancer progression.

\section{Exosomes, NLRP3, and Host Defense}

Leishmania parasites infect the macrophages and inhibit the production and release of several pro-inflammatory cytokines, e.g., IL-1 $\beta$ and IL-18 suppress the host defense and hence survive. The critical virulence factor of Leishmania--metalloprotease glycoprotein 63 (GP-63) can exhibit the PKC pathway, which is of vital importance for both ROS generation and the downstream NLRP3 signaling activation (Cosentino-Gomes et al., 2012; Shio et al., 2015). Exosomes contribute a lot as a major transporter of GP-63. GP-63 has been shown to promote the cleavage of a wide variety of cell substrates, of which cell phosphatase is the most evident. Interestingly, TXNIP, which has been shown to promote the activation of ROS-dependent inflammasomes, was identified as one of the GP-63-cleaved proteins (Shio et al., 2015).

Moreover, the endosymbiotic virus Leishmania RNA Virus 1 virus co-infection can potentiate the interference to the NLRP3 inflammasome network induced by exosomal GP-63 (Olivier and Zamboni, 2020). Membrane glycoconjugate lipophosphoglycan from Leishmania parasites stimulates macrophages through caspase-11, therefore, triggering the non-canonical NLRP3 activation, which is conducted in a pro-inflammatory way instead of an immunosuppressive way (de Carvalho et al., 2019). Limited evidence suggested that it might be transported to the host macrophage's cytoplasm by exosomes before or after the Leishmania invasion (Silverman et al., 2010).

According to previous in vivo research, exosomal HIV-1 protein Nef can prone to induce the activation and redistribution of TLR4 in raft lipid (Mukhamedova et al., 2019). Also, the occurrence of subsequent NLRP3 signal cascade and downstream inflammatory response elevation has been confirmed (Mukhamedova et al., 2019). Worm exosomes were associated with NLRP3-mediated IL-18 secretion in gastrointestinal infection (Alhallaf et al., 2018).

\section{Therapeutic Potentials of Exosomes on NLRP3}

Exosomes as carriers for drugs are being actively researched. Compared to liposomes, injected exosomes can effectively invade 
other cells and perform functions with minimal immune elimination (Kalluri, 2016; Fitts et al., 2019). Moreover, exosomes are more promising for therapeutic use as they have proven to be well tolerated. Repeated injections of MSC-derived exosomes did not induce toxicity in mice (Mendt et al., 2018). Repeated MSC-derived exosomes injections were well tolerated and showed no substantial side effects in patients with graftversus-host disease (Kordelas et al., 2014).

By far, some results indicated the potential therapeutic link between exosomes and NLRP3. Injected $\alpha v$ integrin-specific RGD ( $R$, arginine; $G$, glycine; $D$, aspartic acid)-modified peptide on engineered natural exosomal DOX delivery platform exhibited therapeutic response in mammary tumor mice model (Kim et al., 2016). DOX is cytotoxic drug targeting tumors that could induce pyroptosis by activating NLRP3 inflammasomes (Antonopoulos et al., 2013; Zeng et al., 2020; Wu et al., 2021), thus DOX-carried exosomes might exert latent therapeutic effects on regulating the NLRP3 signal pathway. In PD mouse model, after injection, blood-derived exosomes carried with dopamine were detected in the brain. And compared with free dopamine, exosomal dopamine exhibited higher therapeutic efficacy with reduced toxicity. Similarly, dopamine has been proven to control inflammation by inhibiting NLRP3 inflammasome in PD (Yan et al., 2015; Zhu et al., 2018; Cheng et al., 2020).

However, the potential therapeutic application of exosomes on NLRP3 remains to be verified in further studies.

\section{CONCLUSION}

Nearly 40 years have passed since the exosomes were first discovered (Zhang et al., 2019d). Indeed, numerous basic and clinical studies have uncovered the mystery of exosomes under physiologic and pathologic conditions (Kalluri and LeBleu, 2020). Noteworthy, it has been widely recognized that exosomes have a crucial role in many diseases (Yang et al., 2020). The relationship between inflammation and exosomes in diseases has been further explored, highlighting the importance of NLRP3 inflammasome (Cypryk et al., 2018). The subsequent studies have shown that

\section{REFERENCES}

Afonina, I. S., Zhong, Z., Karin, M., and Beyaert, R. (2017). Limiting InflammationThe Negative Regulation of NF-Kb and the NLRP3 Inflammasome. Nat. Immunol. 18, 861-869. doi:10.1038/ni.3772

Alhallaf, R., Agha, Z., Miller, C. M., Robertson, A. A. B., Sotillo, J., Croese, J., et al. (2018). The Nlrp3 Inflammasome Suppresses Protective Immunity to Gastrointestinal Helminth Infection. Cel Rep. 23, 1085-1098. doi:10.1016/ j.celrep.2018.03.097

Andrejeva, G., and Rathmell, J. C. (2017). Similarities and Distinctions of Cancer and Immune Metabolism in Inflammation and Tumors. Cel Metab. 26, 49-70. doi:10.1016/j.cmet.2017.06.004

Antonopoulos, C., El Sanadi, C., Kaiser, W. J., Mocarski, E. S., and Dubyak, G. R. (2013). Proapoptotic Chemotherapeutic Drugs Induce Noncanonical Processing and Release of IL-1 $\beta$ via Caspase- 8 in Dendritic Cells. J.I. 191, 4789-4803. doi:10.4049/jimmunol.1300645 exosomes can modulate NLRP3 inflammasome, thus affecting the diseases process. Exosomal miR-148a can reduce the damage of the ischemic myocardium via the TXNIP-NLRP3-caspase-1 pathway (Vicencio et al., 2015). Other studies indicated that MSC-Exo-derived miR-126 could suppress hyperglycemiainduced inflammation by down-regulating HMGB1, which can bind to TLRs and activate the NLRP3 pathway (Zhang et al., 2019c). Profound insight into the molecular mechanisms underlying the exosome regulation of NLPR3 could promote their application in clinical treatment. In these studies on exosomes modulation, the molecular mechanisms in diseases progression were mainly confirmed by "gain-of-function" experiments, which may not be accurate enough to represent the exact functions of exosomes to the full extent. Meanwhile, the existing literature failed to identify the actual molecular pathway between exosomes and NLRP3. Therefore, the application of exosomes in the treatment of various diseases has great potential and deserves further exploring. We anticipate that more significant research will break the bottlenecks and lead to breakthroughs in clinical treatment.

\section{AUTHOR CONTRIBUTIONS}

Conceptualization, JZ and PY; writing-original draft preparation, ZL, XC, and JT; writing-review and editing, PY and AS; project administration, PY; funding acquisition, JZ and PY. All authors have read and agreed to the published version of the manuscript.

\section{FUNDING}

This research was supported by grants from the Natural Science Foundation in Jiangxi Province grant (No. 202002BAB216022 to ZJ, No. 20192ACBL21037 and No. 202004BCJL23049 to PY), the National Natural Science Foundation of China (No. 81760048 and No. 82160371 to ZJ, No. 81760050 and No. 82100869 to PY).

Antonopoulos, C., Russo, H. M., El Sanadi, C., Martin, B. N., Li, X., Kaiser, W. J., et al. (2015). Caspase-8 as an Effector and Regulator of Nlrp3 Inflammasome Signaling. J. Biol. Chem. 290, 20167-20184. doi:10.1074/jbc.M115.652321

Atay, S., Gercel-Taylor, C., and Taylor, D. D. (2011). Human Trophoblast-Derived Exosomal Fibronectin Induces Pro-inflammatory Il-1 $\beta$ Production by Macrophages. Am. J. Reprod. Immunol. 66, 259-269. doi:10.1111/j.16000897.2011.00995.x

Aucher, A., Rudnicka, D., and Davis, D. M. (2013). Micrornas Transfer from Human Macrophages to Hepato-Carcinoma Cells and Inhibit Proliferation. J.I. 191, 6250-6260. doi:10.4049/jimmunol.1301728

Bai, L., Li, J., Li, H., Song, J., Zhou, Y., Lu, R., et al. (2019). Renoprotective Effects of Artemisinin and Hydroxychloroquine Combination Therapy on IgA Nephropathy via Suppressing NF-Kb Signaling and NLRP3 Inflammasome Activation by Exosomes in Rats. Biochem. Pharmacol. 169, 113619. doi:10.1016/j.bcp.2019.08.021

Bardi, G. T., Smith, M. A., and Hood, J. L. (2018). Melanoma Exosomes Promote Mixed M1 and M2 Macrophage Polarization. Cytokine 105, 63-72. doi:10.1016/ j.cyto.2018.02.002 
Bauernfeind, F. G., Horvath, G., Stutz, A., Alnemri, E. S., MacDonald, K., Speert, D., et al. (2009). Cutting Edge: NF-Kb Activating Pattern Recognition and Cytokine Receptors License NLRP3 Inflammasome Activation by Regulating NLRP3 Expression. J. Immunol. 183, 787-791. doi:10.4049/jimmunol.0901363

Becatti, M., Taddei, N., Cecchi, C., Nassi, N., Nassi, P. A., and Fiorillo, C. (2012). Sirt1 Modulates Mapk Pathways in Ischemic-Reperfused Cardiomyocytes. Cell. Mol. Life Sci. 69, 2245-2260. doi:10.1007/s00018-012-0925-5

Bechard, M. E., Smalling, R., Hayashi, A., Zhong, Y., Word, A. E., Campbell, S. L., et al. (2020). Pancreatic Cancers Suppress Negative Feedback of Glucose Transport to Reprogram Chromatin for Metastasis. Nat. Commun. 11, 4055. doi:10.1038/s41467-020-17839-5

Bossaller, L., Chiang, P.-I., Schmidt-Lauber, C., Ganesan, S., Kaiser, W. J., Rathinam, V. A. K., et al. (2012). Cutting Edge: FAS (CD95) Mediates Noncanonical IL-1 $\beta$ and IL-18 Maturation via Caspase-8 in an RIP3independent Manner. J.I. 189, 5508-5512. doi:10.4049/jimmunol.1202121

Bouchareychas, L., Duong, P., Covarrubias, S., Alsop, E., Phu, T. A., Chung, A., et al. (2020). Macrophage Exosomes Resolve Atherosclerosis by Regulating Hematopoiesis and Inflammation via Microrna Cargo. Cel Rep. 32, 107881. doi:10.1016/j.celrep.2020.107881

Bowie, R. V., Donatello, S., Lyes, C., Owens, M. B., Babina, I. S., Hudson, L., et al. (2012). Lipid Rafts Are Disrupted in Mildly Inflamed Intestinal Microenvironments without Overt Disruption of the Epithelial Barrier. Am. J. Physiology-Gastrointestinal Liver Physiol. 302, G781-G793. doi:10.1152/ ajpgi.00002.2011

Bruchard, M., Mignot, G., Derangère, V., Chalmin, F., Chevriaux, A., Végran, F., et al. (2013). Chemotherapy-triggered Cathepsin B Release in Myeloid-Derived Suppressor Cells Activates the Nlrp3 Inflammasome and Promotes Tumor Growth. Nat. Med. 19, 57-64. doi:10.1038/nm.2999

Cai, X., Chen, J., Xu, H., Liu, S., Jiang, Q.-X., Halfmann, R., et al. (2014). Prion-like Polymerization Underlies Signal Transduction in Antiviral Immune Defense and Inflammasome Activation. Cell 156, 1207-1222. doi:10.1016/ j.cell.2014.01.063

Cai, X., Zhang, Z.-y., Yuan, J.-t., Ocansey, D. K. W., Tu, Q., Zhang, X., et al. (2021). Hucmsc-derived Exosomes Attenuate Colitis by Regulating Macrophage Pyroptosis via the Mir-378a-5p/nlrp3 axis. Stem Cel Res Ther 12, 416. doi:10.1186/s13287-021-02492-6

Chaput, N., Taïeb, J., André, F., and Zitvogel, L. (2005). The Potential of Exosomes in Immunotherapy. Expert Opin. Biol. Ther. 5, 737-747. doi:10.1517/ 14712598.5.6.737

Chen, P., Huang, Y., Bong, R., Ding, Y., Song, N., Wang, X., et al. (2011). Tumorassociated Macrophages Promote Angiogenesis and Melanoma Growth via Adrenomedullin in a Paracrine and Autocrine Manner. Clin. Cancer Res. 17, 7230-7239. doi:10.1158/1078-0432.Ccr-11-1354

Chen, R.-J., Lee, Y.-H., Chen, T.-H., Chen, Y.-Y., Yeh, Y.-L., Chang, C.-P., et al. (2021). Carbon Monoxide-Triggered Health Effects: The Important Role of the Inflammasome and its Possible Crosstalk with Autophagy and Exosomes. Arch. Toxicol. 95, 1141-1159. doi:10.1007/s00204-021-02976-7

Cheng, J., Liao, Y., Dong, Y., Hu, H., Yang, N., Kong, X., et al. (2020). Microglial Autophagy Defect Causes Parkinson Disease-like Symptoms by Accelerating Inflammasome Activation in Mice. Autophagy 16, 2193-2205. doi:10.1080/ 15548627.2020.1719723

Console, L., Scalise, M., and Indiveri, C. (2019). Exosomes in Inflammation and Role as Biomarkers. Clinica Chim. Acta 488, 165-171. doi:10.1016/ j.cca.2018.11.009

Cosentino-Gomes, D., Rocco-Machado, N., and Meyer-Fernandes, J. R. (2012). Cell Signaling through Protein Kinase C Oxidation and Activation. Ijms 13, 10697-10721. doi:10.3390/ijms130910697

Cypryk, W., Nyman, T. A., and Matikainen, S. (2018). From Inflammasome to Exosome-Does Extracellular Vesicle Secretion Constitute an Inflammasomedependent Immune Response?. Front. Immunol. 9, 2188. doi:10.3389/ fimmu.2018.02188https://www.ncbi.nlm.nih.gov/pmc/articles/PMC6167409/ pdf/fimmu-09-02188.pdf

Dai, Y., Wang, S., Chang, S., Ren, D., Shali, S., Li, C., et al. (2020). M2 MacrophageDerived Exosomes Carry microRNA-148a to Alleviate Myocardial Ischemia/ reperfusion Injury via Inhibiting TXNIP and the TLR4/NF-Kb/nlrp3 Inflammasome Signaling Pathway. J. Mol. Cell Cardiol. 142, 65-79. doi:10.1016/j.yjmcc.2020.02.007 de Carvalho, R. V. H., Andrade, W. A., Lima-Junior, D. S., Dilucca, M., de Oliveira, C. V., Wang, K., et al. (2019). Leishmania Lipophosphoglycan Triggers Caspase-11 and the Non-canonical Activation of the Nlrp3 Inflammasome. Cel Rep. 26, 429-437.e5. doi:10.1016/j.celrep.2018.12.047

Deb, A., Gupta, S., and Mazumder, P. B. (2021). Exosomes: A New Horizon in Modern Medicine. Life Sci. 264, 118623. doi:10.1016/j.lfs.2020.118623

Dinarello, C. A. (2009). Immunological and Inflammatory Functions of the Interleukin-1 Family. Annu. Rev. Immunol. 27, 519-550. doi:10.1146/ annurev.immunol.021908.132612

Ding, J., Wang, K., Liu, W., She, Y., Sun, Q., Shi, J., et al. (2016). Pore-forming Activity and Structural Autoinhibition of the Gasdermin Family. Nature 535, 111-116. doi:10.1038/nature18590

Dmitrieva-Posocco, O., Dzutsev, A., Posocco, D. F., Hou, V., Yuan, W., Thovarai, V., et al. (2019). Cell-type-specific Responses to Interleukin-1 Control Microbial Invasion and Tumor-Elicited Inflammation in Colorectal Cancer. Immunity 50, 166-180.e7. doi:10.1016/j.immuni.2018.11.015

Downs, K. P., Nguyen, H., Dorfleutner, A., and Stehlik, C. (2020). An Overview of the Non-canonical Inflammasome. Mol. Aspects Med. 76, 100924. doi:10.1016/ j.mam.2020.100924

Doyle, L., and Wang, M. (2019). Overview of Extracellular Vesicles, Their Origin, Composition, Purpose, and Methods for Exosome Isolation and Analysis. Cells 8, 727. doi:10.3390/cells 8070727

Dubyak, G. R. (2012). P2x7 Receptor Regulation of Non-classical Secretion from Immune Effector Cells. Cell Microbiol 14, 1697-1706. doi:10.1111/cmi.12001

Elliott, E. I., and Sutterwala, F. S. (2015). Initiation and Perpetuation of Nlrp3 Inflammasome Activation and Assembly. Immunol. Rev. 265, 35-52. doi:10.1111/imr.12286

Erozenci, L. A., Böttger, F., Bijnsdorp, I. V., and Jimenez, C. R. (2019). Urinary Exosomal Proteins as (pan-)cancer Biomarkers: Insights from the Proteome. FEBS Lett. 593, 1580-1597. doi:10.1002/1873-3468.13487

Fitts, C. A., Ji, N., Li, Y., and Tan, C. (2019). Exploiting Exosomes in Cancer Liquid Biopsies and Drug Delivery. Adv. Healthc. Mater. 8, 1801268. doi:10.1002/ adhm.201801268

Fujita, K., and Nonomura, N. (2018). Urinary Biomarkers of Prostate Cancer. Int. J. Urol. 25, 770-779. doi:10.1111/iju.13734

Gemel, J., Kilkus, J., Dawson, G., and Beyer, E. (2019). Connecting Exosomes and Connexins. Cancers 11, 476. doi:10.3390/cancers11040476

Gong, Z., Pan, J., Shen, Q., Li, M., and Peng, Y. (2018). Mitochondrial Dysfunction Induces Nlrp3 Inflammasome Activation during Cerebral Ischemia/ reperfusion Injury. J. Neuroinflammation 15, 242. doi:10.1186/s12974-018$1282-6$

Greening, D. W., Gopal, S. K., Xu, R., Simpson, R. J., and Chen, W. (2015). Exosomes and Their Roles in Immune Regulation and Cancer. Semin. Cel Dev. Biol. 40, 72-81. doi:10.1016/j.semcdb.2015.02.009

Gritsenko, A., Yu, S., Martin-Sanchez, F., Diaz-Del-Olmo, I., Nichols, E.-M., Davis, D. M., et al. (2020). Priming Is Dispensable for Nlrp3 Inflammasome Activation in Human Monocytes In Vitro. Front. Immunol. 11, 565924. doi:10.3389/ fimmu.2020.565924

Ham, S., Lima, L. G., Chai, E. P. Z., Muller, A., Lobb, R. J., Krumeich, S., et al. (2018). Breast Cancer-Derived Exosomes Alter Macrophage Polarization via Gp130/stat3 Signaling. Front. Immunol. 9, 871. doi:10.3389/ fimmu.2018.00871

Haneklaus, M., Gerlic, M., O’Neill, L. A. J., and Masters, S. L. (2013). Mir-223: Infection, Inflammation and Cancer. J. Intern. Med. 274, 215-226. doi:10.1111/ joim. 12099

He, Y., Hara, H., and Núñez, G. (2016). Mechanism and Regulation of Nlrp3 Inflammasome Activation. Trends Biochem. Sci. 41, 1012-1021. doi:10.1016/ j.tibs.2016.09.002

He, Y., Zeng, M. Y., Yang, D., Motro, B., and Núñez, G. (2016). Nek7 Is an Essential Mediator of Nlrp3 Activation Downstream of Potassium Efflux. Nature 530, 354-357. doi:10.1038/nature16959

Huang, S., Ge, X., Yu, J., Han, Z., Yin, Z., Li, Y., et al. (2018). Increased miR-124-3p in Microglial Exosomes Following Traumatic Brain Injury Inhibits Neuronal Inflammation and Contributes to Neurite Outgrowthviatheir Transfer into Neurons. FASEB j. 32, 512-528. doi:10.1096/fj.201700673R

Huang, Y., Xu, W., and Zhou, R. (2021). Nlrp3 Inflammasome Activation and Cell Death. Cell Mol Immunol 18, 2114-2127. doi:10.1038/s41423-021-00740-6 
Ibrahim, A., and Marbán, E. (2016). Exosomes: Fundamental Biology and Roles in Cardiovascular Physiology. Annu. Rev. Physiol. 78, 67-83. doi:10.1146/ annurev-physiol-021115-104929

Incalza, M. A., D’Oria, R., Natalicchio, A., Perrini, S., Laviola, L., and Giorgino, F. (2018). Oxidative Stress and Reactive Oxygen Species in Endothelial Dysfunction Associated with Cardiovascular and Metabolic Diseases. Vasc. Pharmacol. 100, 1-19. doi:10.1016/j.vph.2017.05.005

Jia, X., Qiu, T., Yao, X., Jiang, L., Wang, N., Wei, S., et al. (2020). Arsenic Induces Hepatic Insulin Resistance via Mtros-Nlrp3 Inflammasome Pathway. J. Hazard. Mater. 399, 123034. doi:10.1016/j.jhazmat.2020.123034

Jia, Y., Chen, Y., Wang, Q., Jayasinghe, U., Luo, X., Wei, Q., et al. (2017). Exosome: Emerging Biomarker in Breast Cancer. Oncotarget 8, 41717-41733. doi:10.18632/oncotarget.16684

Jia, Y., Cui, R., Wang, C., Feng, Y., Li, Z., Tong, Y., et al. (2020). Metformin Protects against Intestinal Ischemia-Reperfusion Injury and Cell Pyroptosis via TxnipNlrp3-Gsdmd Pathway. Redox Biol. 32, 101534. doi:10.1016/ j.redox.2020.101534

Jiang, K., Yang, J., Guo, S., Zhao, G., Wu, H., and Deng, G. (2019). Peripheral Circulating Exosome-Mediated Delivery of Mir-155 as a Novel Mechanism for Acute Lung Inflammation. Mol. Ther. 27, 1758-1771. doi:10.1016/ j.ymthe.2019.07.003

Jo, E.-K., Kim, J. K., Shin, D.-M., and Sasakawa, C. (2016). Molecular Mechanisms Regulating Nlrp3 Inflammasome Activation. Cel Mol Immunol 13, 148-159. doi:10.1038/cmi.2015.95

Ju, S., Mu, J., Dokland, T., Zhuang, X., Wang, Q., Jiang, H., et al. (2013). Grape Exosome-like Nanoparticles Induce Intestinal Stem Cells and Protect Mice from Dss-Induced Colitis. Mol. Ther. 21, 1345-1357. doi:10.1038/mt.2013.64

Kalluri, R., and LeBleu, V. S. (2020). The Biology, Function, and Biomedical Applications of Exosomes. Science 367, eaau6977. doi:10.1126/science.aau6977

Kalluri, R. (2016). The Biology and Function of Exosomes in Cancer. J. Clin. Invest. 126, 1208-1215. doi:10.1172/jci81135

Kang, M., Choi, J. K., Jittayasothorn, Y., and Egwuagu, C. E. (2020). Interleukin 35producing Exosomes Suppress Neuroinflammation and Autoimmune Uveitis. Front. Immunol. 11, 1051. doi:10.3389/fimmu.2020.01051

Karmakar, M., Katsnelson, M. A., Dubyak, G. R., and Pearlman, E. (2016). Neutrophil P2X7 Receptors Mediate NLRP3 Inflammasome-dependent IL$1 \beta$ Secretion in Response to ATP. Nat. Commun. 7, 10555. doi:10.1038/ ncomms 10555

Kim, M. S., Haney, M. J., Zhao, Y., Mahajan, V., Deygen, I., Klyachko, N. L., et al. (2016). Development of Exosome-Encapsulated Paclitaxel to Overcome Mdr in Cancer Cells. Nanomedicine: Nanotechnology, Biol. Med. 12, 655-664. doi:10.1016/j.nano.2015.10.012

Kim, Y.-B., Ahn, Y.-H., Jung, J.-H., Lee, Y.-J., Lee, J.-H., and Kang, J. L. (2019). Programming of Macrophages by Uv-Irradiated Apoptotic Cancer Cells Inhibits Cancer Progression and Lung Metastasis. Cel Mol Immunol 16, 851-867. doi:10.1038/s41423-019-0209-1

Koh, E., Lee, E. J., Nam, G.-H., Hong, Y., Cho, E., Yang, Y., et al. (2017). ExosomeSirpa, a CD47 Blockade Increases Cancer Cell Phagocytosis. Biomaterials 121, 121-129. doi:10.1016/j.biomaterials.2017.01.004

Koka, S., Xia, M., Chen, Y., Bhat, O. M., Yuan, X., Boini, K. M., et al. (2017). Endothelial Nlrp3 Inflammasome Activation and Arterial Neointima Formation Associated with Acid Sphingomyelinase during Hypercholesterolemia. Redox Biol. 13, 336-344. doi:10.1016/ j.redox.2017.06.004

Komada, T., and Muruve, D. A. (2019). The Role of Inflammasomes in Kidney Disease. Nat. Rev. Nephrol. 15, 501-520. doi:10.1038/s41581-019-0158-z

Kordelas, L., Rebmann, V., Ludwig, A.-K., Radtke, S., Ruesing, J., Doeppner, T. R., et al. (2014). Msc-derived Exosomes: A Novel Tool to Treat Therapy-Refractory Graft-Versus-Host Disease. Leukemia 28, 970-973. doi:10.1038/leu.2014.41

Kore, R. A., Wang, X., Ding, Z., Griffin, R. J., Tackett, A. J., and Mehta, J. L. (2021). Msc Exosome-Mediated Cardioprotection in Ischemic Mouse Heart Comparative Proteomics of Infarct and Peri-Infarct Areas. Mol. Cel Biochem 476, 1691-1704. doi:10.1007/s11010-020-04029-6

Lamkanfi, M., and Dixit, V. M. (2012). Inflammasomes and Their Roles in Health and Disease. Annu. Rev. Cel Dev. Biol. 28, 137-161. doi:10.1146/annurevcellbio-101011-155745

Lerner, A. G., Upton, J.-P., Praveen, P. V. K., Ghosh, R., Nakagawa, Y., Igbaria, A., et al. (2012). IRE1 $\alpha$ Induces Thioredoxin-Interacting Protein to Activate the
NLRP3 Inflammasome and Promote Programmed Cell Death under Irremediable ER Stress. Cel Metab. 16, 250-264. doi:10.1016/j.cmet.2012.07.007 Li, G., Huang, D., Li, N., Ritter, J. K., and Li, P.-L. (2021). Regulation of Trpml1 Channel Activity and Inflammatory Exosome Release by Endogenously Produced Reactive Oxygen Species in Mouse Podocytes. Redox Biol. 43, 102013. doi:10.1016/j.redox.2021.102013

Li, H., Huang, X., Chang, X., Yao, J., He, Q., Shen, Z., et al. (2020). S100-A9 Protein in Exosomes Derived from Follicular Fluid Promotes Inflammation via Activation of NF-kB Pathway in Polycystic Ovary Syndrome. J. Cel Mol Med 24, 114-125. doi: $10.1111 / \mathrm{jcmm} .14642$

Li, X., Lei, Y., Wu, M., and Li, N. (2018). Regulation of Macrophage Activation and Polarization by Hcc-Derived Exosomal Lncrna Tuc339. Ijms 19, 2958. doi:10.3390/ijms19102958

Liang, M., Chen, X., Wang, L., Qin, L., Wang, H., Sun, Z., et al. (2020). Cancerderived Exosomal Trim59 Regulates Macrophage Nlrp3 Inflammasome Activation to Promote Lung Cancer Progression. J. Exp. Clin. Cancer Res. 39, 176. doi:10.1186/s13046-020-01688-7

Liu, D., Zeng, X., Li, X., Mehta, J. L., and Wang, X. (2018). Role of Nlrp3 Inflammasome in the Pathogenesis of Cardiovascular Diseases. Basic Res. Cardiol. 113, 5. doi:10.1007/s00395-017-0663-9

Liu, X., Zhang, Z., Ruan, J., Pan, Y., Magupalli, V. G., Wu, H., et al. (2016). Inflammasome-activated Gasdermin D Causes Pyroptosis by Forming Membrane Pores. Nature 535, 153-158. doi:10.1038/nature18629

Liu, Y., Lou, G., Li, A., Zhang, T., Qi, J., Ye, D., et al. (2018). Amsc-derived Exosomes Alleviate Lipopolysaccharide/d-Galactosamine-Induced Acute Liver Failure by Mir-17-Mediated Reduction of Txnip/nlrp3 Inflammasome Activation in Macrophages. EBioMedicine 36, 140-150. doi:10.1016/ j.ebiom.2018.08.054

Liu, Y., Wang, R., Jiang, J., Yang, B., Cao, Z., and Cheng, X. (2015). Mir-223 Is Upregulated in Monocytes from Patients with Tuberculosis and Regulates Function of Monocyte-Derived Macrophages. Mol. Immunol. 67, 475-481. doi:10.1016/j.molimm.2015.08.006

Liu, Z., Wang, C., Yang, J., Chen, Y., Zhou, B., Abbott, D. W., et al. (2020). Caspase1 Engages Full-Length Gasdermin D through Two Distinct Interfaces that Mediate Caspase Recruitment and Substrate Cleavage. Immunity 53, 106-114.e5. doi:10.1016/j.immuni.2020.06.007

Lu, A., Li, Y., Schmidt, F. I., Yin, Q., Chen, S., Fu, T.-M., et al. (2016). Molecular Basis of Caspase-1 Polymerization and its Inhibition by a New Capping Mechanism. Nat. Struct. Mol. Biol. 23, 416-425. doi:10.1038/nsmb.3199

Lu, A., Magupalli, V. G., Ruan, J., Yin, Q., Atianand, M. K., Vos, M. R., et al. (2014). Unified Polymerization Mechanism for the Assembly of Asc-dependent Inflammasomes. Cell 156, 1193-1206. doi:10.1016/j.cell.2014.02.008

Ludwig, A.-K., and Giebel, B. (2012). Exosomes: Small Vesicles Participating in Intercellular Communication. Int. J. Biochem. Cel Biol. 44, 11-15. doi:10.1016/ j.biocel.2011.10.005

Ludwig, N., Whiteside, T. L., and Reichert, T. E. (2019). Challenges in Exosome Isolation and Analysis in Health and Disease. Ijms 20, 4684. doi:10.3390/ ijms20194684

Mangan, M. S. J., Olhava, E. J., Roush, W. R., Seidel, H. M., Glick, G. D., and Latz, E. (2018). Targeting the Nlrp3 Inflammasome in Inflammatory Diseases. Nat. Rev. Drug Discov. 17, 588-606. doi:10.1038/nrd.2018.97

Mao, Q., Liang, X.-L., Zhang, C.-L., Pang, Y.-H., and Lu, Y.-X. (2019). Lncrna Klf3-As1 in Human Mesenchymal Stem Cell-Derived Exosomes Ameliorates Pyroptosis of Cardiomyocytes and Myocardial Infarction through Mir-138-5p/sirt1 axis. Stem Cel Res Ther 10, 393. doi:10.1186/ s13287-019-1522-4

Masumoto, J., Taniguchi, S. i., Ayukawa, K., Sarvotham, H., Kishino, T., Niikawa, N., et al. (1999). Asc, a Novel 22-kda Protein, Aggregates during Apoptosis of Human Promyelocytic Leukemia Hl-60 Cells. J. Biol. Chem. 274, 33835-33838. doi:10.1074/jbc.274.48.33835

Mendt, M., Kamerkar, S., Sugimoto, H., McAndrews, K. M., Wu, C.-C., Gagea, M., et al. (2018). Generation and Testing of Clinical-Grade Exosomes for Pancreatic Cancer. JCI Insight 3, e99263. doi:10.1172/jci.insight.99263

Miao, E. A., Alpuche-Aranda, C. M., Dors, M., Clark, A. E., Bader, M. W., Miller, S. I., et al. (2006). Cytoplasmic Flagellin Activates Caspase-1 and Secretion of Interleukin $1 \beta$ via Ipaf. Nat. Immunol. 7, 569-575. doi:10.1038/ni1344

Minutoli, L., Puzzolo, D., Rinaldi, M., Irrera, N., Marini, H., Arcoraci, V., et al. (2016). Ros-mediated Nlrp3 Inflammasome Activation in Brain, Heart, Kidney, 
and Testis Ischemia/reperfusion Injury. Oxidative Med. Cell Longevity 2016, 1-10. doi:10.1155/2016/2183026

Mohamed, I. N., Hafez, S. S., Fairaq, A., Ergul, A., Imig, J. D., and El-Remessy, A. B. (2014). Thioredoxin-interacting Protein Is Required for Endothelial Nlrp3 Inflammasome Activation and Cell Death in a Rat Model of High-Fat Diet. Diabetologia 57, 413-423. doi:10.1007/s00125-013-3101-z

Moossavi, M., Parsamanesh, N., Bahrami, A., Atkin, S. L., and Sahebkar, A. (2018). Role of the Nlrp3 Inflammasome in Cancer. Mol. Cancer 17, 158. doi:10.1186/ s12943-018-0900-3

Mukhamedova, N., Hoang, A., Dragoljevic, D., Dubrovsky, L., Pushkarsky, T., Low, H., et al. (2019). Exosomes Containing Hiv Protein Nef Reorganize Lipid Rafts Potentiating Inflammatory Response in Bystander Cells. Plos Pathog. 15, e1007907. doi:10.1371/journal.ppat.1007907

Nabhan, J. F., Hu, R., Oh, R. S., Cohen, S. N., and Lu, Q. (2012). Formation and Release of Arrestin Domain-Containing Protein 1-mediated Microvesicles (Armms) at Plasma Membrane by Recruitment of Tsg101 Protein. Proc. Natl. Acad. Sci. 109, 4146-4151. doi:10.1073/pnas.1200448109

Njock, M.-S., Guiot, J., Henket, M. A., Nivelles, O., Thiry, M., Dequiedt, F., et al. (2019). Sputum Exosomes: Promising Biomarkers for Idiopathic Pulmonary Fibrosis. Thorax 74, 309-312. doi:10.1136/thoraxjnl-2018-211897

Noonin, C., and Thongboonkerd, V. (2021). Exosome-inflammasome Crosstalk and Their Roles in Inflammatory Responses. Theranostics 11, 4436-4451. doi:10.7150/thno.54004

Oh, K., Lee, O.-Y., Park, Y., Seo, M. W., and Lee, D.-S. (2016). IL-1 $\beta$ Induces IL-6 Production and Increases Invasiveness and Estrogen-independent Growth in a TG2-dependent Manner in Human Breast Cancer Cells. BMC Cancer 16, 724. doi:10.1186/s12885-016-2746-7

Olivier, M., and Zamboni, D. S. (2020). Leishmania Viannia Guyanensis, Lrv1 Virus and Extracellular Vesicles: A Dangerous Trio Influencing the faith of Immune Response during Muco-Cutaneous Leishmaniasis. Curr. Opin. Immunol. 66, 108-113. doi:10.1016/j.coi.2020.08.004

Orning, P., Lien, E., and Fitzgerald, K. A. (2019). Gasdermins and Their Role in Immunity and Inflammation. J. Exp. Med. 216, 2453-2465. doi:10.1084/ jem.20190545

Pajukanta, P., Lilja, H. E., Sinsheimer, J. S., Cantor, R. M., Lusis, A. J., Gentile, M., et al. (2004). Familial Combined Hyperlipidemia Is Associated with Upstream Transcription Factor 1 (Usf1). Nat. Genet. 36, 371-376. doi:10.1038/ng1320

Pearce, L., Davidson, S. M., and Yellon, D. M. (2021). Does Remote Ischaemic Conditioning Reduce Inflammation? A Focus on Innate Immunity and Cytokine Response. Basic Res. Cardiol. 116, 12. doi:10.1007/s00395-02100852-0

Pegtel, D. M., and Gould, S. J. (2019). Exosomes. Annu. Rev. Biochem. 88, 487-514. doi:10.1146/annurev-biochem-013118-111902

Poggio, M., Hu, T., Pai, C.-C., Chu, B., Belair, C. D., Chang, A., et al. (2019). Suppression of Exosomal Pd-L1 Induces Systemic Anti-tumor Immunity and Memory. Cell 177, 414-427.e13. doi:10.1016/j.cell.2019.02.016

Próchnicki, T., and Latz, E. (2017). Inflammasomes on the Crossroads of Innate Immune Recognition and Metabolic Control. Cel Metab. 26, 71-93. doi:10.1016/j.cmet.2017.06.018

Qu, Y., Ramachandra, L., Mohr, S., Franchi, L., Harding, C. V., Nunez, G., et al. (2009). P2x7 Receptor-Stimulated Secretion of Mhc Class Ii-Containing Exosomes Requires the Asc/nlrp3 Inflammasome but Is Independent of Caspase-1. J. Immunol. 182, 5052-5062. doi:10.4049/jimmunol.0802968

Raposo, G., and Stoorvogel, W. (2013). Extracellular Vesicles: Exosomes, Microvesicles, and Friends. J. Cel Biol 200, 373-383. doi:10.1083/jcb.201211138

Rathinam, V. A. K., Zhao, Y., and Shao, F. (2019). Innate Immunity to Intracellular Lps. Nat. Immunol. 20, 527-533. doi:10.1038/s41590-019-0368-3

Ridker, P. M., Everett, B. M., Thuren, T., MacFadyen, J. G., Chang, W. H., Ballantyne, C., et al. (2017). Antiinflammatory Therapy with Canakinumab for Atherosclerotic Disease. N. Engl. J. Med. 377, 1119-1131. doi:10.1056/ NEJMoa1707914

Saberi, M., Woods, N.-B., de Luca, C., Schenk, S., Lu, J. C., Bandyopadhyay, G., et al. (2009). Hematopoietic Cell-specific Deletion of Toll-like Receptor 4 Ameliorates Hepatic and Adipose Tissue Insulin Resistance in High-Fat-Fed Mice. Cel Metab. 10, 419-429. doi:10.1016/j.cmet.2009.09.006

Sarkar, S., Rokad, D., Malovic, E., Luo, J., Harischandra, D. S., Jin, H., et al. (2019). Manganese Activates Nlrp3 Inflammasome Signaling and Propagates Exosomal
Release of Asc in Microglial Cells. Sci. Signal. 12, eaat9900. doi:10.1126/ scisignal.aat 9900

Schroder, K., Zhou, R., and Tschopp, J. (2010). The Nlrp3 Inflammasome: A Sensor for Metabolic Danger?. Science 327, 296-300. doi:10.1126/science.1184003

Seoane, P. I., Lee, B., Hoyle, C., Yu, S., Lopez-Castejon, G., Lowe, M., et al. (2020). The Nlrp3-Inflammasome as a Sensor of Organelle Dysfunction. J. Cel Biol 219. doi:10.1083/jcb.202006194

Shang, S., Ji, X., Zhang, L., Chen, J., Li, C., Shi, R., et al. (2019). Macrophage ABHD5 Suppresses NF-kb-dependent Matrix Metalloproteinase Expression and Cancer Metastasis. Cancer Res. 79, canres.1059.2019-26. doi:10.1158/0008-5472.Can19-1059

Sharif, H., Wang, L., Wang, W. L., Magupalli, V. G., Andreeva, L., Qiao, Q., et al. (2019). Structural Mechanism for Nek7-Licensed Activation of Nlrp3 Inflammasome. Nature 570, 338-343. doi:10.1038/s41586-019-1295-z

Sharma, A., and Johnson, A. (2020). Exosome DNA: Critical Regulator of Tumor Immunity and a Diagnostic Biomarker. J. Cel Physiol 235, 1921-1932. doi:10.1002/jcp. 29153

Sharma, B. R., and Kanneganti, T.-D. (2021). Nlrp3 Inflammasome in Cancer and Metabolic Diseases. Nat. Immunol. 22, 550-559. doi:10.1038/s41590-02100886-5

Shi, H., Wang, Y., Li, X., Zhan, X., Tang, M., Fina, M., et al. (2016). Nlrp3 Activation and Mitosis Are Mutually Exclusive Events Coordinated by Nek7, a New Inflammasome Component. Nat. Immunol. 17, 250-258. doi:10.1038/ ni. 3333

Shi, J., Zhao, Y., Wang, Y., Gao, W., Ding, J., Li, P., et al. (2014). Inflammatory Caspases Are Innate Immune Receptors for Intracellular Lps. Nature 514, 187-192. doi:10.1038/nature13683

Shi, X., Xie, X., Sun, Y., He, H., Huang, H., Liu, Y., et al. (2020). Paeonol Inhibits Nlrp3 Mediated Inflammation in Rat Endothelial Cells by Elevating Hyperlipidemic Rats Plasma Exosomal Mirna-223. Eur. J. Pharmacol. 885, 173473. doi:10.1016/j.ejphar.2020.173473

Shio, M. T., Christian, J. G., Jung, J. Y., Chang, K.-P., and Olivier, M. (2015). Pkc/ ros-mediated Nlrp3 Inflammasome Activation Is Attenuated by Leishmania Zinc-Metalloprotease during Infection. Plos Negl. Trop. Dis. 9, e0003868. doi:10.1371/journal.pntd.0003868

Si, X.-L., Fang, Y.-J., Li, L.-F., Gu, L.-Y., Yin, X.-Z., Jun-Tian, T., et al. (2021). From Inflammasome to Parkinson's Disease: Does the Nlrp3 Inflammasome Facilitate Exosome Secretion and Exosomal Alpha-Synuclein Transmission in Parkinson's Disease?. Exp. Neurol. 336, 113525. doi:10.1016/ j.expneurol.2020.113525

Silverman, J. M., Clos, J., Horakova, E., Wang, A. Y., Wiesgigl, M., Kelly, I., et al. (2010). Leishmania Exosomes Modulate Innate and Adaptive Immune Responses through Effects on Monocytes and Dendritic Cells. J.I. 185, 5011-5022. doi:10.4049/jimmunol.1000541

Simons, M., and Raposo, G. (2009). Exosomes - Vesicular Carriers for Intercellular Communication. Curr. Opin. Cel Biol. 21, 575-581. doi:10.1016/ j.ceb.2009.03.007

Singla, D., Johnson, T., and Tavakoli Dargani, Z. (2019). Exosome Treatment Enhances Anti-inflammatory M2 Macrophages and Reduces InflammationInduced Pyroptosis in Doxorubicin-Induced Cardiomyopathy. Cells 8, 1224. doi:10.3390/cells8101224

Strowig, T., Henao-Mejia, J., Elinav, E., and Flavell, R. (2012). Inflammasomes in Health and Disease. Nature 481, 278-286. doi:10.1038/nature10759

Subramanian, N., Natarajan, K., Clatworthy, M. R., Wang, Z., and Germain, R. N. (2013). The Adaptor Mavs Promotes Nlrp3 Mitochondrial Localization and Inflammasome Activation. Cell 153, 348-361. doi:10.1016/j.cell.2013.02.054

Sutterwala, F. S., Ogura, Y., Szczepanik, M., Lara-Tejero, M., Lichtenberger, G. S., Grant, E. P., et al. (2006). Critical Role for Nalp3/cias1/cryopyrin in Innate and Adaptive Immunity through its Regulation of Caspase-1. Immunity 24, 317-327. doi:10.1016/j.immuni.2006.02.004

Swanson, K. V., Deng, M., and Ting, J. P.-Y. (2019). The Nlrp3 Inflammasome: Molecular Activation and Regulation to Therapeutics. Nat. Rev. Immunol. 19, 477-489. doi:10.1038/s41577-019-0165-0

Tai, Y. L., Chen, K. C., Hsieh, J. T., and Shen, T. L. (2018). Exosomes in Cancer Development and Clinical Applications. Cancer Sci. 109, 2364-2374. doi:10.1111/cas.13697

Tang, J., Jin, L., Liu, Y., Li, L., Ma, Y., Lu, L., et al. (2020). Exosomes Derived from Mesenchymal Stem Cells Protect the Myocardium against Ischemia/ 
reperfusion Injury through Inhibiting Pyroptosis. Dddt Vol. 14, 3765-3775. doi: $10.2147 /$ dddt.S239546

Tao, Y., Wang, N., Qiu, T., and Sun, X. (2020). The Role of Autophagy and Nlrp3 Inflammasome in Liver Fibrosis. Biomed. Res. Int. 2020, 1-8. doi:10.1155/2020/ 7269150

Tavakoli Dargani, Z., and Singla, D. K. (2019). Embryonic Stem Cell-Derived Exosomes Inhibit Doxorubicin-Induced Tlr4-Nlrp3-Mediated Cell DeathPyroptosis. Am. J. Physiology-Heart Circulatory Physiol. 317, H460-H471. doi:10.1152/ajpheart.00056.2019

Ting, J. P.-Y., Lovering, R. C., Alnemri, E. S., Bertin, J., Boss, J. M., Davis, B. K., et al. (2008). The Nlr Gene Family: A Standard Nomenclature. Immunity 28, 285-287. doi:10.1016/j.immuni.2008.02.005

Tschopp, J., and Schroder, K. (2010). Nlrp3 Inflammasome Activation: The Convergence of Multiple Signalling Pathways on Ros Production?. Nat. Rev. Immunol. 10, 210-215. doi:10.1038/nri2725

van Niel, G., D'Angelo, G., and Raposo, G. (2018). Shedding Light on the Cell Biology of Extracellular Vesicles. Nat. Rev. Mol. Cel Biol 19, 213-228. doi:10.1038/nrm.2017.125

Vandanmagsar, B., Youm, Y.-H., Ravussin, A., Galgani, J. E., Stadler, K., Mynatt, R. L., et al. (2011). The Nlrp3 Inflammasome Instigates Obesity-Induced Inflammation and Insulin Resistance. Nat. Med. 17, 179-188. doi:10.1038/ nm.2279

Vicencio, J. M., Yellon, D. M., Sivaraman, V., Das, D., Boi-Doku, C., Arjun, S., et al. (2015). Plasma Exosomes Protect the Myocardium from Ischemia-Reperfusion Injury. J. Am. Coll. Cardiol. 65, 1525-1536. doi:10.1016/ j.jacc.2015.02.026https://www.ncbi.nlm.nih.gov/pubmed/25881934

Wan, X., Xu, C., Yu, C., and Li, Y. (20162016). Role of Nlrp3 Inflammasome in the Progression of Nafld to Nash. Can. J. Gastroenterol. Hepatol. 2016, 1-7. doi:10.1155/2016/6489012

Wang, J., Liu, Y., Sun, W., Zhang, Q., Gu, T., and Li, G. (2018). Plasma Exosomes as Novel Biomarker for the Early Diagnosis of Gastric Cancer. Cbm 21, 805-812. doi:10.3233/cbm-170738

Wang, L., and Hauenstein, A. V. (2020). The Nlrp3 Inflammasome: Mechanism of Action, Role in Disease and Therapies. Mol. Aspects Med. 76, 100889. doi:10.1016/j.mam.2020.100889https://www.ncbi.nlm.nih.gov/pubmed/ 32859386

Wang, W., Hu, D., Feng, Y., Wu, C., Song, Y., Liu, W., et al. (2020). Paxillin Mediates Atp-Induced Activation of P2x7 Receptor and Nlrp3 Inflammasome. BMC Biol. 18, 182. doi:10.1186/s12915-020-00918-w

Wang, X., Jiang, W., Yan, Y., Gong, T., Han, J., Tian, Z., et al. (2014). Rna Viruses Promote Activation of the Nlrp3 Inflammasome through a Rip1Rip3-Drp1 Signaling Pathway. Nat. Immunol. 15, 1126-1133. doi:10.1038/ni.3015

Wang, Y., Xie, W., Liu, B., Huang, H., Luo, W., Zhang, Y., et al. (2021). Stem CellDerived Exosomes Repair Ischemic Muscle Injury by Inhibiting the Tumor Suppressor Rb1-Mediated Nlrp3 Inflammasome Pathway. Sig Transduct Target. Ther. 6, 121. doi:10.1038/s41392-021-00520-8

Weber, A. N. R., Bittner, Z. A., Shankar, S., Liu, X., Chang, T.-H., Jin, T., et al. (2020). Recent Insights into the Regulatory Networks of Nlrp3 Inflammasome Activation. J. Cel Sci 133, jcs248344. doi:10.1242/jcs.248344

Willeit, P., Ridker, P. M., Nestel, P. J., Simes, J., Tonkin, A. M., Pedersen, T. R., et al. (2018). Baseline and On-Statin Treatment Lipoprotein(a) Levels for Prediction of Cardiovascular Events: Individual Patient-Data Meta-Analysis of Statin Outcome Trials. The Lancet 392, 1311-1320. doi:10.1016/s0140-6736(18) 31652-0

Wu, Q., Li, W., Zhao, J., Sun, W., Yang, Q., Chen, C., et al. (2021). Apigenin Ameliorates Doxorubicin-Induced Renal Injury via Inhibition of Oxidative Stress and Inflammation. Biomed. Pharmacother. 137, 111308. doi:10.1016/ j.biopha.2021.111308

$\mathrm{Wu}, \mathrm{T}$., Hong, Y., Jia, L., Wu, J., Xia, J., Wang, J., et al. (2016). Modulation of IL-1 $\beta$ Reprogrammes the Tumor Microenvironment to Interrupt Oral Carcinogenesis. Sci. Rep. 6, 20208. doi:10.1038/srep20208

Xia, Y., Chen, S., Zhu, G., Huang, R., Yin, Y., and Ren, W. (2018). Betaine Inhibits Interleukin- $1 \beta$ Production and Release: Potential Mechanisms. Front. Immunol. 9, 2670. doi:10.3389/fimmu.2018.02670

Xu, Z., Zeng, S., Gong, Z., and Yan, Y. (2020). Exosome-based Immunotherapy: A Promising Approach for Cancer Treatment. Mol. Cancer 19, 160. doi:10.1186/ s12943-020-01278-3
Xue, Y., Enosi Tuipulotu, D., Tan, W. H., Kay, C., and Man, S. M. (2019). Emerging Activators and Regulators of Inflammasomes and Pyroptosis. Trends Immunol. 40, 1035-1052. doi:10.1016/j.it.2019.09.005

Xue, Z., Zhang, Z., Liu, H., Li, W., Guo, X., Zhang, Z., et al. (2019). Lincrnacox2 Regulates Nlrp3 Inflammasome and Autophagy Mediated Neuroinflammation. Cell Death Differ 26, 130-145. doi:10.1038/ s41418-018-0105-8

Yan, B., Zhang, Y., Liang, C., Liu, B., Ding, F., Wang, Y., et al. (2020). Stem CellDerived Exosomes Prevent Pyroptosis and Repair Ischemic Muscle Injury through a Novel Exosome/circhipk3/Foxo3a Pathway. Theranostics 10, 6728-6742. doi:10.7150/thno.42259

Yan, Y., Jiang, W., Liu, L., Wang, X., Ding, C., Tian, Z., et al. (2015). Dopamine Controls Systemic Inflammation through Inhibition of Nlrp3 Inflammasome. Cell 160, 62-73. doi:10.1016/j.cell.2014.11.047

Yang, D., Zhang, W., Zhang, H., Zhang, F., Chen, L., Ma, L., et al. (2020). Progress, Opportunity, and Perspective on Exosome Isolation - Efforts for Efficient ExosomeBased Theranostics. Theranostics 10, 3684-3707. doi:10.7150/thno.41580

Yang, T. T., Liu, C. G., Gao, S. C., Zhang, Y., and Wang, P. C. (2018). The Serum Exosome Derived Microrna-135a, -193b, and -384 Were Potential Alzheimer's Disease Biomarkers. Biomed. Environ. Sci. 31, 87-96. doi: $10.3967 /$ bes2018.011

Yang, Y., Li, C.-W., Chan, L.-C., Wei, Y., Hsu, J.-M., Xia, W., et al. (2018). Exosomal Pd-L1 Harbors Active Defense Function to Suppress T Cell Killing of Breast Cancer Cells and Promote Tumor Growth. Cell Res 28, 862-864. doi:10.1038/s41422-018-0060-4https:// www.ncbi.nlm.nih.gov/pubmed/29959401

Yang, Y., Wang, H., Kouadir, M., Song, H., and Shi, F. (2019). Recent Advances in the Mechanisms of Nlrp3 Inflammasome Activation and its Inhibitors. Cell Death Dis 10, 128. doi:10.1038/s41419-019-1413-8

Yuan, D., Zhao, Y., Banks, W. A., Bullock, K. M., Haney, M., Batrakova, E., et al. (2017). Macrophage Exosomes as Natural Nanocarriers for Protein Delivery to Inflamed Brain. Biomaterials 142, 1-12. doi:10.1016/ j.biomaterials.2017.07.011

Zamboni, D. S., and Lima-Junior, D. S. (2015). Inflammasomes in Host Response to Protozoan Parasites. Immunol. Rev. 265, 156-171. doi:10.1111/imr.12291

Zeng, C., Duan, F., Hu, J., Luo, B., Huang, B., Lou, X., et al. (2020). Nlrp3 Inflammasome-Mediated Pyroptosis Contributes to the Pathogenesis of Non-ischemic Dilated Cardiomyopathy. Redox Biol. 34, 101523. doi:10.1016/ j.redox.2020.101523

Zhang, A., Wang, G., Jia, L., Su, T., and Zhang, L. (2019). Exosome-mediated microRNA-138 and Vascular Endothelial Growth Factor in Endometriosis through Inflammation and Apoptosis via the Nuclear Factor-Kb Signaling Pathway. Int. J. Mol. Med. 43, 358-370. doi:10.3892/ijmm.2018.3980

Zhang, H., Wang, L., Li, C., Yu, Y., Yi, Y., Wang, J., et al. (2019). Exosome-induced Regulation in Inflammatory Bowel Disease. Front. Immunol. 10, 1464. doi:10.3389/fimmu.2019.01464

Zhang, L., Li, Z., Skrzypczynska, K. M., Fang, Q., Zhang, W., O’Brien, S. A., et al. (2020). Single-cell Analyses Inform Mechanisms of Myeloid-Targeted Therapies in colon Cancer. Cell 181, 442-459.e29. doi:10.1016/j.cell.2020.03.048

Zhang, L., Wang, Q., Su, H., and Cheng, J. (2021). Exosomes from Adipose Derived Mesenchymal Stem Cells Alleviate Diabetic Osteoporosis in Rats through Suppressing Nlrp3 Inflammasome Activation in Osteoclasts. J. Biosci. Bioeng. 131, 671-678. doi:10.1016/j.jbiosc.2021.02.007

Zhang, L., and Yu, D. (2019). Exosomes in Cancer Development, Metastasis, and Immunity. Biochim. Biophys. Acta (Bba) - Rev. Cancer 1871, 455-468. doi:10.1016/j.bbcan.2019.04.004

Zhang, M., Hu, G., Shao, N., Qin, Y., Chen, Q., Wang, Y., et al. (2021). Thioredoxin-interacting Protein (Txnip) as a Target for Alzheimer's Disease: Flavonoids and Phenols. Inflammopharmacol 29, 1317-1329. doi:10.1007/s10787-021-00861-4

Zhang, S., Teo, K. Y. W., Chuah, S. J., Lai, R. C., Lim, S. K., and Toh, W. S. (2019). Msc Exosomes Alleviate Temporomandibular Joint Osteoarthritis by Attenuating Inflammation and Restoring Matrix Homeostasis. Biomaterials 200, 35-47. doi:10.1016/j.biomaterials.2019.02.006

Zhang, W., Wang, Y., and Kong, Y. (2019). Exosomes Derived from Mesenchymal Stem Cells Modulate Mir-126 to Ameliorate Hyperglycemia-Induced Retinal Inflammation via Targeting Hmgb1. Invest. Ophthalmol. Vis. Sci. 60, 294-303. doi:10.1167/iovs.18-25617 
Zhang, X., Jiang, Y., Huang, Q., Wu, Z., Pu, H., Xu, Z., et al. (2021). Exosomes Derived from Adipose-Derived Stem Cells Overexpressing Glyoxalase-1 Protect Endothelial Cells and Enhance Angiogenesis in Type 2 Diabetic Mice with Limb Ischemia. Stem Cel Res Ther 12, 403. doi:10.1186/s13287-021-02475-7

Zhen, Y., and Zhang, H. (2019). Nlrp3 Inflammasome and Inflammatory Bowel Disease. Front. Immunol. 10, 276. doi:10.3389/fimmu.2019.00276

Zhong, Z., Umemura, A., Sanchez-Lopez, E., Liang, S., Shalapour, S., Wong, J., et al. (2016). NF- $\kappa B$ Restricts Inflammasome Activation via Elimination of Damaged Mitochondria. Cell 164, 896-910. doi:10.1016/j.cell.2015.12.057

Zhou, R., Tardivel, A., Thorens, B., Choi, I., and Tschopp, J. (2010). Thioredoxininteracting Protein Links Oxidative Stress to Inflammasome Activation. Nat. Immunol. 11, 136-140. doi:10.1038/ni.1831

Zhou, W., Chen, C., Chen, Z., Liu, L., Jiang, J., Wu, Z., et al. (2018). Nlrp3: A Novel Mediator in Cardiovascular Disease. J. Immunol. Res. 2018, 1-8. doi:10.1155/2018/ 5702103

Zhou, X., Brown, B. A., Siegel, A. P., El Masry, M. S., Zeng, X., Song, W., et al. (2020). Exosome-mediated Crosstalk between Keratinocytes and Macrophages in Cutaneous Wound Healing. ACS Nano 14, 12732-12748. doi:10.1021/ acsnano.0c03064

Zhou, Y., Tong, Z., Jiang, S., Zheng, W., Zhao, J., and Zhou, X. (2020). The Roles of Endoplasmic Reticulum in Nlrp3 Inflammasome Activation. Cells 9, 1219. doi:10.3390/cells9051219
Zhu, J., Hu, Z., Han, X., Wang, D., Jiang, Q., Ding, J., et al. (2018). Dopamine D2 Receptor Restricts Astrocytic NLRP3 Inflammasome Activation via Enhancing the Interaction of $\beta$-arrestin2 and NLRP3. Cel Death Differ 25, 2037-2049. doi:10.1038/s41418-018-0127-2

Conflict of Interest: The authors declare that the research was conducted in the absence of any commercial or financial relationships that could be construed as a potential conflict of interest.

Publisher's Note: All claims expressed in this article are solely those of the authors and do not necessarily represent those of their affiliated organizations, or those of the publisher, the editors and the reviewers. Any product that may be evaluated in this article, or claim that may be made by its manufacturer, is not guaranteed or endorsed by the publisher.

Copyright (c) $2022 \mathrm{Li}$, Chen, Tao, Shi, Zhang and Yu. This is an open-access article distributed under the terms of the Creative Commons Attribution License (CC BY). The use, distribution or reproduction in other forums is permitted, provided the original author(s) and the copyright owner(s) are credited and that the original publication in this journal is cited, in accordance with accepted academic practice. No use, distribution or reproduction is permitted which does not comply with these terms. 


\section{GLOSSARY}

ABHD5 abhydrolase domain containing 5

ASC apoptosis-associated speck-like protein containing a CARD

ATP adenosine triphosphate

CARD c-terminal caspase recruitment domain

CD47 cluster of differentiation 47

CD63 cluster of differentiation 63

CD81 cluster of differentiation 81

CD9 cluster of differentiation 9

circHIPK3 circular homeodomain-interacting protein kinase 3circular homeodomain-interacting protein kinase 3

DAMPs damage associated molecular patterns

DOX doxorubicin

Drp1 dynamin-related protein 1

EEF1A1 recombinant eukaryotic translation elongation factor 1 alpha 1

EEF2 recombinant eukaryotic translation elongation factor 2

ES-Exo embryonic stem cell-derived exosomes

EVs extracellular vesicles

FABP5 fatty acid-binding protein 5

FOXO3a fork head box class O 3a

GELNs ginger ELNs

GP-63 glycoprotein 63

GSDMD gasdermin D

HMGB1 high mobility group box-1

Hsp heat shock proteins

I/R ischemia/reperfusion

IL-18 interleukin-18

IL-1 $\beta$ interleukin-1 $\beta$

ILVs intraluminal vesicles

IPF idiopathic pulmonary fibrosis

LLNs liposome-like nanoparticles

lncRNA long non-coding RNA

LPS lipopolysaccharides

LRR leucine-rich repeat domain
MAVS mitochondrial abtiviral-signaling protein

MHC major histocompatibility complex

miRNAs microRNAs

mRNAs messenger RNAs

MSC mesenchymal stem cell

mtROS mitochondrial reactive oxygen species

MVB multivesicular bodies

NACHT nucleotide-binding and oligomerization domain

NASH non-alcoholic steatohepatitis

NCMs neonatal rat cardiomyocytes

NEK7 NIMA related kinase 7

NF- $\mathbf{k B}$ nuclear factor $\kappa \mathrm{B}$

NLR NOD-like receptor

NLRP3 NOD-like receptor family, pyrin domain containing 3

ox-mtDNA oxidized mitochondrial DNA

P2X7R purinergic ligand-gated ion channel 7 receptor

PAMPs pathogen-associated molecular patterns

PC prostate cancer

PD Parkinson's disease

PD1 programmed death-1

PD-L1 programmed death-ligand 1

PPAR peroxisome proliferator-activated receptor

PTEN phosphate and tension homology deleted on chromsome ten

PYD N-terminal pyrin domain

ROS reactive oxygen species

S100-A9 S100 calcium binding protein A9

SIRPa signal regulatory protein $a$

SIRT1 sirtuin 1

TAMs tumor-associated macrophages

TLRs toll-like receptors

TME tumor microenvironment

TRIM59 Tripartite motif-containing 59

TXNIP thioredoxin-interacting protein

UMSC-Exo umbilical cord mesenchymal stem cells-derived exosomes 